\title{
Non-parametric deprojection of NIKA SZ observations: Pressure distribution in the Planck-discovered cluster PSZ1 G045.85+57.71
}

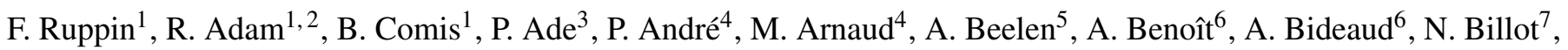
O. Bourrion ${ }^{1}$, M. Calvo ${ }^{6}$, A. Catalano ${ }^{1}$, G. Coiffard ${ }^{8}$, A. D'Addabbo ${ }^{6,9}$, M. De Petris ${ }^{9}$, F.-X. Désert ${ }^{10}$, S. Doyle ${ }^{3}$, J. Goupy ${ }^{6}$, C. Kramer ${ }^{7}$, S. Leclercq ${ }^{8}$, J. F. Macías-Pérez ${ }^{1}$, P. Mauskopf ${ }^{3,11}$, F. Mayet ${ }^{1}$, A. Monfardini ${ }^{6}$, F. Pajot ${ }^{5}$, E. Pascale ${ }^{3}$, L. Perotto ${ }^{1}$, G. Pisano ${ }^{3}$, E. Pointecouteau ${ }^{12,13}$, N. Ponthieu ${ }^{10}$, G. W. Pratt ${ }^{4}$, V. Revéret ${ }^{4}$, A. Ritacco ${ }^{1}$, L. Rodriguez ${ }^{4}$, C. Romero ${ }^{8}$, K. Schuster ${ }^{8}$, A. Sievers ${ }^{7}$, S. Triqueneaux ${ }^{6}$, C. Tucker ${ }^{3}$, and R. Zylka ${ }^{8}$

${ }^{1}$ Laboratoire de Physique Subatomique et de Cosmologie, Université Grenoble Alpes, CNRS/IN2P3, 53 avenue des Martyrs, Grenoble, France e-mail: ruppin@lpsc.in2p3.fr

2 Laboratoire Lagrange, Université Côte d'Azur, Observatoire de la Côte d'Azur, CNRS, Bd de l'Observatoire, CS 34229, 06304 Nice Cedex 4, France

3 Astronomy Instrumentation Group, University of Cardiff, UK

${ }^{4}$ Laboratoire AIM, CEA/IRFU, CNRS/INSU, Université Paris Diderot, CEA-Saclay, 91191 Gif-Sur-Yvette, France

${ }_{6}^{5}$ Institut d'Astrophysique Spatiale (IAS), CNRS and Université Paris Sud, Orsay, France

6 Institut Néel, CNRS and Université Grenoble Alpes, France

7 Institut de RadioAstronomie Millimétrique (IRAM), Granada, Spain

8 Institut de RadioAstronomie Millimétrique (IRAM), Grenoble, France

9 Dipartimento di Fisica, Sapienza Università di Roma, Piazzale Aldo Moro 5, 00185 Roma, Italy

${ }^{10}$ Institut de Planétologie et d'Astrophysique de Grenoble (IPAG), CNRS and Université Grenoble Alpes, France

11 School of Earth and Space Exploration and Department of Physics, Arizona State University, Tempe, AZ 85287, USA

12 Université de Toulouse, UPS-OMP, Institut de Recherche en Astrophysique et Planétologie (IRAP), 31028 Toulouse, France

13 CNRS, IRAP, 9 avenue Colonel Roche, BP 44346, 31028 Toulouse Cedex 4, France

Received 26 July 2016 / Accepted 10 September 2016

\begin{abstract}
The determination of the thermodynamic properties of clusters of galaxies at intermediate and high redshift can bring new insights into the formation of large-scale structures. It is essential for a robust calibration of the mass-observable scaling relations and their scatter, which are key ingredients for precise cosmology using cluster statistics. Here we illustrate an application of high resolution $(<20$ arcsec) thermal Sunyaev-Zel'dovich (tSZ) observations by probing the intracluster medium (ICM) of the Planck-discovered galaxy cluster PSZ1 G045.85+57.71 at redshift $z=0.61$, using tSZ data obtained with the NIKA camera, which is a dual-band $(150$ and $260 \mathrm{GHz})$ instrument operated at the IRAM 30-m telescope. We deproject jointly NIKA and Planck data to extract the electronic pressure distribution from the cluster core $\left(R \sim 0.02 R_{500}\right)$ to its outskirts $\left(R \sim 3 R_{500}\right)$ non-parametrically for the first time at intermediate redshift. The constraints on the resulting pressure profile allow us to reduce the relative uncertainty on the integrated Compton parameter by a factor of two compared to the Planck value. Combining the tSZ data and the deprojected electronic density profile from XMM-Newton allows us to undertake a hydrostatic mass analysis, for which we study the impact of a spherical model assumption on the total mass estimate. We also investigate the radial temperature and entropy distributions. These data indicate that PSZ1 G045.85+57.71 is a massive $\left(M_{500} \sim 5.5 \times 10^{14} M_{\odot}\right)$ cool-core cluster. This work is part of a pilot study aiming at optimizing the treatment of the NIKA2 tSZ large program dedicated to the follow-up of SZ-discovered clusters at intermediate and high redshifts. This study illustrates the potential of NIKA2 to put constraints on the thermodynamic properties and tSZ-scaling relations of these clusters, and demonstrates the excellent synergy between tSZ and X-ray observations of similar angular resolution.
\end{abstract}

Key words. galaxies: clusters: intracluster medium - instrumentation: high angular resolution - cosmology: observations

\section{Introduction}

Galaxy clusters are the ultimate manifestation of the hierarchical structure formation process in the standard cosmological model, and as such, they are sensitive to both the matter content and expansion history of the Universe in which they form. Clusters are thus potentially powerful tools to infer cosmological parameters. In particular, counting clusters as a function of their mass and redshift (e.g., Sehgal et al. 2011; Planck Collaboration XXIV 2016; de Haan et al. 2016) brings constraints on the cosmological parameters that are complementary to those derived with other probes such as type Ia supernovae (e.g., Riess et al. 2007), the CMB temperature and polarization angular power spectra (e.g., Planck Collaboration XIII 2016), or baryonic acoustic oscillations (e.g., Anderson et al. 2014).

About $85 \%$ of the total mass in galaxy clusters is from dark matter. The principal baryonic component is found in the hot, ionized, X-ray emitting gas of the intracluster medium (ICM), containing about $12 \%$ of the total mass. The remaining baryonic 
mass is found in the stellar population. Cluster masses can be inferred from several independent observables. The velocity dispersion of the galaxies (e.g., Biviano et al. 2006; Sifón et al. 2016), various X-ray properties such as temperature or luminosity (e.g., Vikhlinin et al. 2006; Pratt et al. 2009), or the lensing distortions of background galaxies (e.g., Applegate et al. 2014; Umetsu et al. 2014; Hoekstra et al. 2015) can be related to the underlying total mass. Another observational probe of interest is the thermal Sunyaev-Zel'dovich effect (tSZ; Sunyaev \& Zeldovich 1972), which is due to the inverse Compton scattering of cosmic microwave background (CMB) photons with high-energy electrons of the ICM. As this effect is directly proportional to the thermal energy contained in the ICM, it is expected to provide a low scatter mass proxy for galaxy clusters (e.g., da Silva et al. 2004; Nagai et al. 2007a). Furthermore, as the tSZ effect is a CMB spectral distortion, it does not suffer from cosmological dimming. This observable is therefore a powerful probe to estimate both galaxy cluster total mass and baryonic content distribution up to high redshift.

The Planck satellite, the South Pole Telescope (SPT), and the Atacama Cosmology Telescope (ACT) surveys have used tSZ observations to discover and characterize large galaxy cluster samples (e.g., Planck Collaboration XXVII 2016; Bleem et al. 2015; Hasselfield et al. 2013). In addition, individual observations of known clusters have been obtained with a number of instruments, such as APEX-SZ, CARMA, SZA, BOLOCAM, and AMIs (e.g., Schwan et al. 2011; Plagge et al. 2013; Muchovej et al. 2007; Sayers et al. 2012; Perrott et al. 2015). However, their relatively low angular resolution ( $>1$ arcmin) restricts the tSZ characterization of the ICM to low redshift (Plagge et al. 2010; Basu et al. 2010; Bonamente et al. 2012; Planck Collaboration Int. V 2013; Sayers et al. 2013), as a combination with higher resolution X-ray observations is needed to map clusters at both large and small scales (e.g., Planck Collaboration Int. V 2013; Eckert et al. 2013).

In combination with local data, high angular resolution tSZ observations at intermediate to high-redshift $(z>0.5)$ have a number of different applications. They can be used to study the evolution of structural properties such as cluster pressure profiles and their scatter. Furthermore, they provide new insights and constraints on scaling properties such as the relation between the integrated Compton parameter and the cluster total mass and its scatter. High angular resolution tSZ observations can also be used to characterize the two-dimensional (2D) pressure distribution within the ICM. This information is essential for understanding cluster formation physics and performing precise cosmological analysis with the cluster population.

Cluster growth and evolution is characterized by complex astrophysical phenomena, including deviation from equilibrium and generation of turbulence due to merging events and feedback from active galactic nuclei. While stochastic, the frequency of these events evolves with time and increases at high redshift. They are the prime cause of scatter and deviations from self-similarity in the scaling relations that are used to link observables to mass in cosmological analyses (e.g., Yu et al. 2015; Sembolini et al. 2014). Of particular importance is the clarification of the physical origin of this normalization and scatter in the scaling relations, rendering the use of galaxy clusters for cosmological application more robust.

The New IRAM KIDs Array (NIKA; Monfardini et al. 2011; Bourrion et al. 2012; Calvo et al. 2013) was a dual-band continuum camera operated at the Institut de Radio Astronomie Millimetrique (IRAM) $30 \mathrm{~m}$ telescope between 2010 and 2015. It was one of the very few tSZ instruments with sub-arcminute resolution. Other examples include the Goddard-IRAM Superconducting 2-mm Observer (GISMO; Staguhn et al. 2008) and the Multiplexed SQUID TES array at Ninety Gigahertz (MUSTANG; Korngut et al. 2011). NIKA was the only dual-band subarcminute instrument (Catalano et al. 2014) that observed the tSZ effect simultaneously at 150 and $260 \mathrm{GHz}$ with an angular resolution of 18.2 and 12.0 arcsec, respectively. Furthering the characterization of galaxy cluster pressure profiles that has been initiated by arcminute resolution instruments at low redshift, NIKA has now mapped the pressure distribution in a number of galaxy clusters at intermediate and high redshift (see Adam et al. 2014, 2015, 2016, 2017).

In this paper we detail the NIKA observations of the Planckdiscovered cluster PSZ1 G045.85+57.71 at $z=0.61$. A key result is the first non-parametric measurement with high statistical precision of the pressure profile of a distant cluster at an angular resolution $\sim 20$ arcsec, extending to much higher redshift pioneering non-parametric pressure profile measurements at low resolution (Basu et al. 2010). Basu et al. 2010 have applied the deprojection method presented in Nord et al. 2009 to the APEXSZ data (Halverson et al. 2009) of the nearby cluster Abell 2204 $(z=0.15)$. They have shown that a non-parametric modeling of the gas pressure profile can be obtained. Previous works have shown that deprojection methods can be used to probe the ICM of clusters from simulations (Puchwein et al. 2008; Lee \& Suto 2004; Ameglio et al. 2007).

The work presented in this paper is a pilot study for the forthcoming SZ observations (see Comis et al. 2016) with NIKA2 (see Catalano et al. 2016). The combination with Planck data allows the determination of the non-parametric pressure profile out to scales of $\gtrsim 3 \mathrm{Mpc}$, substantially improving the constraints on the spherically integrated Compton parameter. Using the deprojected gas density profile from XMM-Newton, we reconstruct the thermodynamic properties of the ICM without making use of $\mathrm{X}$-ray spectroscopic information. This result illustrates the excellent synergy between tSZ and X-ray observations of similar angular resolution, and serves as a pilot study for combining tSZ data to measure the gas pressure with short X-ray observations to measure the gas density.

This paper is organized as follows. The NIKA observations of PSZ1 G045.85+57.71 at the IRAM 30-m telescope and the raw data processing are explained in Sect. 2. Ancillary data, previous tSZ observations, point source contamination, and $X M M-N e w t o n$ data reduction, are described in Sect. 3. The modelization of the ICM and the method to estimate the cluster total mass are presented in Sect. 4. We also discuss the characterization of the cluster ellipticity and its impact on the mass estimation. In Sect. 5 a non-parametric multiprobe analysis is performed to extract the radial pressure profile and obtain the ICM thermodynamic properties. The conclusions and NIKA2 perspectives are discussed in Sect. 6. Throughout this study we assume a flat $\Lambda \mathrm{CDM}$ cosmology following the latest Planck results (Planck Collaboration XIII 2016): $H_{0}=67.8 \mathrm{~km} \mathrm{~s}^{-1} \mathrm{Mpc}^{-1}$, $\Omega_{\mathrm{m}}=0.308$, and $\Omega_{\Lambda}=0.692$. Within this framework, at the cluster redshift, one arcsec corresponds to $6.93 \mathrm{kpc}$.

\section{Observation at the IRAM 30-m telescope with NIKA}

We present in this section the NIKA observations of the PSZ1 G045.85+57.71 cluster, performed in October 2014, which have been used to produce the tSZ surface brightness maps at $150 \mathrm{GHz}$ and $260 \mathrm{GHz}$. To begin with, we describe the key elements of the thermal SZ effect. 


\subsection{The thermal Sunyaev-Zel'dovich effect}

The thermal Sunyaev-Zel'dovich effect corresponds to the Compton scattering of CMB photons on high-energy ICM electrons. The scattering equation describing this interaction was derived by Kompaneets (1956) in the case where the radiation temperature is negligible compared to the plasma temperature. Using this equation, Sunyaev \& Zeldovich (1972, 1980) computed the induced variation of the spectral radiance compared to the CMB variation as follows:

$\frac{\Delta I_{\mathrm{tSZ}}}{I_{0}}=y f\left(v, T_{\mathrm{e}}\right)$,

where $y$ is the Compton parameter that describes the amplitude of the spectral distortion, $f\left(v, T_{\mathrm{e}}\right)$ gives the frequency dependence of the tSZ spectrum, and $T_{\mathrm{e}}$ is the ICM electronic temperature. The Compton parameter is related to the line-of-sight integral of the electronic pressure $P_{\mathrm{e}}$,

$y=\frac{\sigma_{\mathrm{T}}}{m_{\mathrm{e}} c^{2}} \int P_{\mathrm{e}} \mathrm{d} l$,

where $m_{\mathrm{e}}$ is the electron mass, $c$ the speed of light, and $\sigma_{\mathrm{T}}$ the Thomson scattering cross section. The integrated Compton parameter $Y_{\text {tot }}$ is then computed via the aperture photometry performed on the Compton parameter map ${ }^{1}$.

The frequency dependence of the tSZ spectrum is given by the expression (Birkinshaw 1999; Carlstrom et al. 2002)

$f\left(x, T_{\mathrm{e}}\right)=\frac{x^{4} \mathrm{e}^{x}}{\left(\mathrm{e}^{x}-1\right)^{2}}\left(x \operatorname{coth}\left(\frac{x}{2}\right)-4\right)\left(1+\delta_{\mathrm{tSZ}}\left(x, T_{\mathrm{e}}\right)\right)$,

with $x=\frac{h v}{k_{\mathrm{B}} T_{\mathrm{CMB}}}$,

where $h$ and $k_{\mathrm{B}}$ are the Planck and Boltzmann constants, respectively, and $\delta_{\mathrm{tSz}}\left(x, T_{\mathrm{e}}\right)$ corresponds to the relativistic correction, which is non-negligible for plasma temperatures larger than $10 \mathrm{keV}$ (Itoh et al. 1998). We thus notice that the spectral deformation induced by the tSZ effect is completely characterized by the $f$ function and does not depend on the plasma temperature if relativistic corrections are negligible. In this case, $f$ is positive (negative) for frequencies higher (lower) than $217 \mathrm{GHz}$. We therefore expect a negative signal on the $150 \mathrm{GHz}$ NIKA map and a positive signal at $260 \mathrm{GHz}$.

\subsection{Observing conditions, scanning strategy, calibration, and data reduction}

PSZ1 G045.85+57.71 was observed by the NIKA camera simultaneously at $150 \mathrm{GHz}$ and $260 \mathrm{GHz}$ during the second NIKA open pool in November 2014. In this section we present the observation conditions, scanning strategy, calibration procedure, and data reduction method.

The pointing center was chosen to be at (RA, Dec 2000 $)=(15: 18: 20.8,+29: 27: 36.75)$ following the Planck and $X M M-N e w t o n$ observations. All the coordinates in this paper are given in the equinox 2000 system. The mean zenith opacities were measured to be 0.21 and 0.27 at 150 and $260 \mathrm{GHz}$, respectively, and the atmosphere was particularly unstable because of the presence of wind, which induces an increased residual noise on the final map (see Catalano et al. 2014, for details on the

\footnotetext{
1 This definition gives the cylindrical Compton parameter of the cluster up to $5 R_{500}$.
}

Table 1. Instrumental characteristics of NIKA at the IRAM 30-m telescope in November 2014.

\begin{tabular}{ccc}
\hline \hline Observing band & $150 \mathrm{GHz}$ & $260 \mathrm{GHz}$ \\
\hline Gaussian beam model FWHM $(\operatorname{arcsec})$ & 18.2 & 12.0 \\
Field of view (arcmin) & 1.9 & 1.8 \\
Effective number of detectors & 113 & 156 \\
Sensitivity (mJy/beam s ${ }^{1 / 2}$ ) & 12 & 61 \\
Conversion factor $y$-Jy/beam & $-11.1 \pm 1.0$ & $3.4 \pm 0.4$ \\
Pointing errors (arcsec) & $<3$ & $<3$ \\
Calibration uncertainties & $9 \%$ & $11 \%$ \\
\hline
\end{tabular}

opacity measurement procedure with NIKA). The mean elevation of the source was 49 degrees. The effective number of valid detectors was 113 at $150 \mathrm{GHz}$ and 156 at $260 \mathrm{GHz}$ for a field of view of 1.9 and 1.8 arcmin, respectively.

The cluster was mapped using on-the-fly raster scans made by a succession of 19 subscans of 6 arcmin length at constant azimuth or elevation with 10 arcsec steps between subscans. After discarding data affected by high atmospheric instabilities, the overall effective observing time on the cluster is $4.35 \mathrm{~h}$.

We used Uranus as a primary calibrator and the Moreno model (Moreno 2010) to estimate its brightness temperature frequency dependence (see Adam et al. 2014; Adam 2015; and Catalano et al. 2014, for details on the calibration procedure). The primary beam was modeled by a Gaussian function with a full width at half maximum (FWHM) that has been measured to be 18.2 and 12.0 arcsec at 150 and $260 \mathrm{GHz}$, respectively. Using the dispersion of the measured Uranus fluxes and the uncertainty on the Moreno model (accurate to 5\%; see Planck Collaboration VIII 2014), the overall calibration uncertainty is estimated to be 9 and $11 \%$ at 150 and $260 \mathrm{GHz}$, respectively. We estimated the conversion factors from the measured surface brightness to the Compton parameter taking the NIKA bandpass measurements into account. We found the computed values to be $-11.1 \pm 1.0$ and $3.4 \pm 0.4 \mathrm{Jy} /$ beam per unit of Compton parameter accounting for calibration uncertainties at 150 and $260 \mathrm{GHz}$, respectively. The main instrumental characteristics of the NIKA camera during the second open pool are summarized in Table 1.

We follow the raw data reduction method detailed in (Adam et al. 2015). The main steps of the procedure are briefly summarized here. The selection of valid detectors is based on their noise properties and optical responses. We removed glitches in the timelines due to impacts of cosmic rays prior to the analysis. We suppressed fluctuations associated with cryogenic vibrations in the Fourier domain. We removed the atmospheric and electronic correlated noise by subtracting the common-mode templates estimated by averaging the timelines for each array. We flagged the cluster using the signal-to-noise ratio $(\mathrm{S} / \mathrm{N})$ map estimation in an iterative way to avoid ringing and reduce the signal filtering. We estimated the resulting transfer function of the data processing using simulations and this function is fairly constant with a filtering of $\sim 5 \%$ at scales smaller than the NIKA field of view but larger than the beam size. The filtering increases rapidly for larger scales (see Fig. 3 in Adam et al. 2015, as a typical example of the transfer function for this analysis). For each scan, the processed time order information is projected on a pixelized grid for the two NIKA frequencies. The computed scans are eventually coadded using 

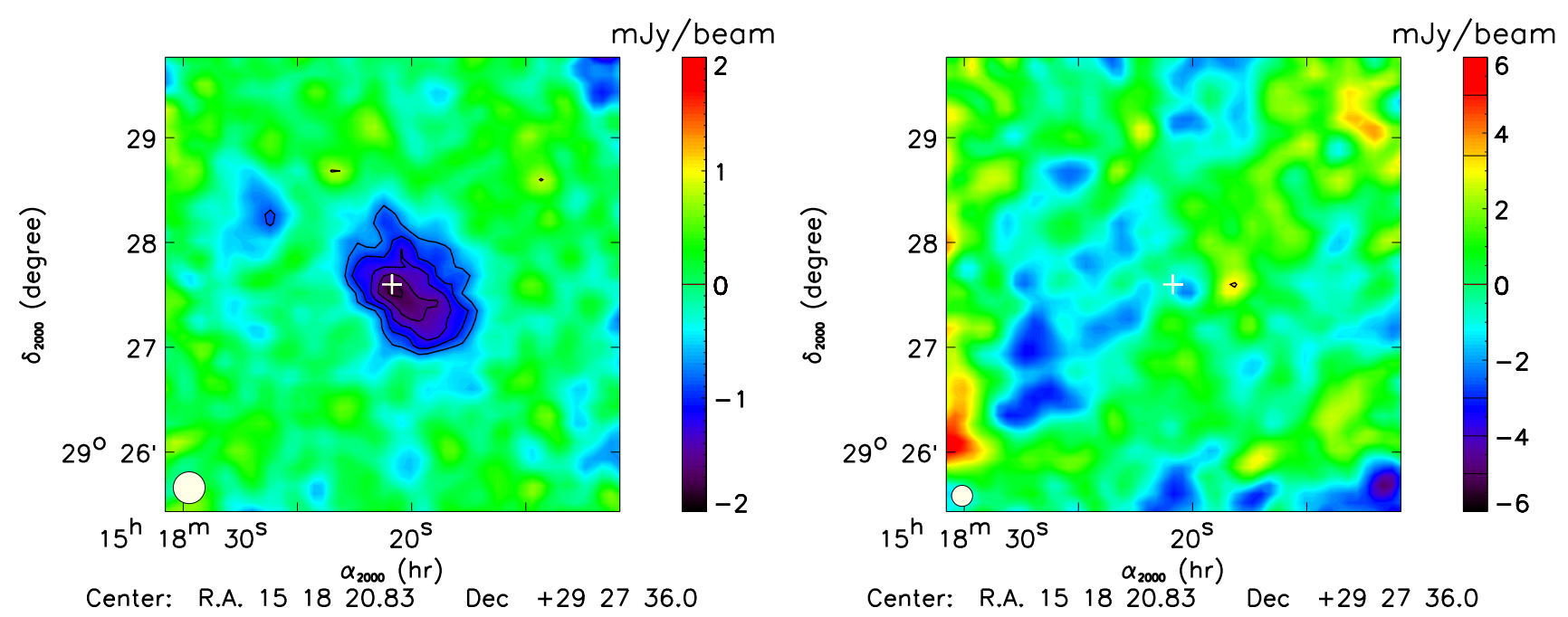

Fig. 1. NIKA tSZ surface brightness maps at $150 \mathrm{GHz}($ left $)$ and $260 \mathrm{GHz}$ (right). The significance of the measured signal is given by the black contours starting at $3 \sigma$ with $1 \sigma$ spacing. The maps are smoothed with an additional $10 \operatorname{arcsec}$ Gaussian filter for display purposes and the NIKA beam FWHMs are represented as white disks in the bottom left-hand corner of the maps. The white crosses indicate the X-ray center. Note that we use the original maps (without additional smoothing) in the following analysis.
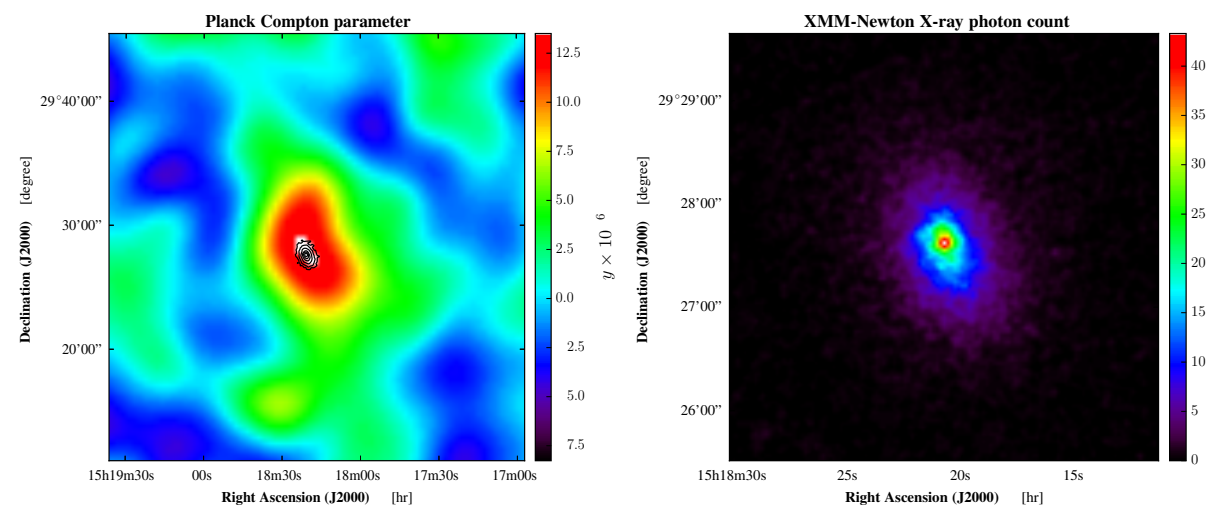

Fig. 2. Left: Planck MILCA Compton parameter map of PSZ1 G045.85+57.71 obtained by extracting a patch of the Planck full sky $y$-map (Planck Collaboration XXII 2016). The $X M M$-Newton X-ray contours are overlaid in black to show the effect of the Planck beam dilution on the cluster inner structure. Right: $X M M$-Newton X-ray photon count map of PSZ1 G045.85+57.71 smoothed with an additional 4 arcsec Gaussian filter for display purposes.

Table 2. Location and flux of the radio sources observed in the $4.4 \times 4.4 \operatorname{arcmin}^{2}$ field around PSZ1 G045.85+57.71.

\begin{tabular}{ccccc}
\hline \hline Source & Identifier & Position & $\begin{array}{c}1.4 \mathrm{GHz} \\
{[\mathrm{mJy}]}\end{array}$ & Reference \\
\hline RS1 & FIRST J151819.5+292712 & $15 \mathrm{~h} 18 \mathrm{~m} 19.5 \mathrm{~s}+29 \mathrm{~d} 27 \mathrm{~m} 13 \mathrm{~s}$ & $1.71 \pm 0.14$ & FIRST, Becker et al. (1995) \\
RS2 & FIRST J151822.4+292917 & $15 \mathrm{~h} 18 \mathrm{~m} 22.5 \mathrm{~s}+29 \mathrm{~d} 29 \mathrm{~m} 18 \mathrm{~s}$ & $2.9 \pm 0.5$ & FIRST, Becker et al. (1995) \\
\hline
\end{tabular}

inverse variance weighting to obtain the final maps shown in Fig. 1.

\subsection{NIKA observations}

The NIKA tSZ surface brightness maps of PSZ1 G045.85+57.71 at 150 and $260 \mathrm{GHz}$ are shown in Fig. 1. The NIKA maps are centered on the X-ray peak coordinates denoted as a white cross and were smoothed with a 10 arcsec Gaussian filter for display purposes. We observe a strong tSZ decrement on the $150 \mathrm{GHz}$ map, which reaches a $7 \sigma$ significance at the surface brightness peak (-1.9 mJy/beam). The observed galaxy cluster exhibits an elliptical morphology with a major axis going from the southwest to the northeast of the center and does not indicate the presence of ICM substructure. Furthermore, the maximum tSZ decrement is aligned with the X-ray peak and thus does not indicate that PSZ1 G045.85+57.71 has a disturbed core.
As expected, there is no significant tSZ signal on the $260 \mathrm{GHz}$ map. Indeed, we can estimate the expected tSZ surface brightness peak at $260 \mathrm{GHz}$ knowing the tSZ surface brightness at $150 \mathrm{GHz}$ and the tSZ spectrum analytic expression (Eq. (3)). The estimated value of $\sim 1 \mathrm{mJy} /$ beam is below the standard deviation of the residual noise in the NIKA $260 \mathrm{GHz}$ map. Furthermore, the $260 \mathrm{GHz}$ map does not present any significant submillimeter point source contamination given the root mean square (rms) noise on this map.

The residual noise on the map has to be characterized to estimate the significance contours of the measured signal at both NIKA frequencies. Furthermore, the noise covariance matrix $C_{\text {NIKA }}$ at $150 \mathrm{GHz}$ has to be estimated to be used for the ICM characterization.

Following the procedure described in (Adam et al. 2016), we use null-map realizations at 150 and $260 \mathrm{GHz}$ to estimate the best-fit noise power spectrum models at both NIKA frequencies. 
The estimated residual noise power spectrum models, together with the integration time per pixel at 150 and $260 \mathrm{GHz}$, enable the simulation of Monte Carlo realizations of noise maps that are used to estimate the $\mathrm{S} / \mathrm{N}$ on the final maps (see Fig. 1) and to compute the noise covariance matrix at $150 \mathrm{GHz}$.

\section{PSZ1 G045.85+57.71 ancillary data}

This section presents the ancillary data obtained from previous observations of PSZ1 G045.85+57.71. These data are used in the following multiprobe ICM characterization and give complementary information on the dynamical state of this cluster.

\subsection{Previous $S Z$ observations of $P S Z 1$ G045.85+57.71}

PSZ1 G045.85+57.71 has been identified by Planck with a $\mathrm{S} / \mathrm{N}$ of 5.06. It is a member of the early Planck SZ catalog (Planck Collaboration XXIX 2014) and its detection has been confirmed in the second catalog release (Planck Collaboration XXVII 2016). The Planck tSZ map of PSZ1 G045.85+57.71 is shown in the left panel of Fig. 2. It has been obtained by extracting a patch of the Planck full sky $y$-map using a Gnomonic projection (Planck Collaboration XXII 2016). The patch used in the ICM analysis is centered on the cluster coordinates and is $1.7 \mathrm{de}-$ gree wide. Its integrated Compton parameter estimated at $R_{500}$ is given in the Planck catalog by $Y_{500}=8.21_{-1.70}^{+1.73} \times 10^{-4}$ arcmin $^{2}$ (Planck Collaboration XXXII 2015) ${ }^{2}$. The corresponding cylindrical integrated Compton parameter at $5 R_{500}$ is given by multiplying this value by 1.79 , when assuming the universal pressure profile of Arnaud et al. (2010). This estimation has been compared with the integrated Compton parameter found by aperture photometry on the Planck $y$-map, $Y_{5 R 500}=1.28 \pm 0.57 \times 10^{-3} \mathrm{arcmin}^{2}$. The error on the estimated value was computed by performing the same aperture photometry measurement on the Planck map randomly around the cluster, where the noise is homogeneous. The estimate of the cluster integrated Compton parameter computed by aperture photometry on the map is therefore compatible with that given in the Planck catalog (Planck Collaboration XXXII 2015). The Planck estimated hydrostatic mass assuming the best-fit $Y$ - $M$ scaling relation of (Arnaud et al. 2010) as a prior was found to be $M_{500}=$ $7.936_{-0.962}^{+0.894} \times 10^{14} M_{\odot}($ Planck Collaboration XXXII 2015). The uncertainties on this cluster mass estimation does not take into account the intrinsic scatter of the scaling relation or systematic errors coming from the data selection for the fit of the scaling relation. A tSZ follow-up of this cluster has been made at $15 \mathrm{GHz}$ by the Arcminute Microkelvin Imager (AMI) at a slightly better resolution ( 3 arcmin compared to the Planck beams of 5-10 arcmin; see Perrott et al. 2015). These AMI observations provide a joint estimation of both the characteristic angular size $\theta_{\mathrm{s}}$ and the integrated Compton parameter $Y_{\text {tot }}$. The latter is equivalent to the Planck estimator $Y_{5 R 500}$ to within 5\% if we assume the universal pressure profile with universal parameter values and the concentration parameter $c_{500}=1.177$ (Arnaud et al. 2010; Perrott et al. 2015). The results derived by AMI are compatible with the Planck results. The combination of both Planck and AMI constraints gives an integrated Compton parameter estimation at $Y_{5 R 500}^{\text {Planck/AMI }}=1.47 \pm 0.51 \times 10^{-3} \mathrm{arcmin}^{2}$ thus improving the Planck estimation by about $10 \%$. This estimation is

\footnotetext{
${ }^{2} R_{\Delta}$ is the radius within which the mean cluster density is equal to $\Delta$ times the critical density of the Universe at the cluster redshift $\rho_{\mathrm{c}}=\frac{3 H(z)^{2}}{8 \pi G}$.
}

used, along with the NIKA data, to give a first estimate of the radial pressure profile of PSZ1 G045.85+57.71.

\subsection{Point source contamination}

As has been shown in Sayers et al. (2013) and Adam et al. (2016), the point source contamination of the tSZ signal has to be studied carefully to avoid significant bias in the ICM characterization. The NRAO VLA Sky Survey (NVSS; Condon et al. 1998) and Faint Images of the Radio Sky at Twenty-Centimeters survey (FIRST; Becker et al. 1995), which cover the northern sky at $1.4 \mathrm{GHz}$, has enabled the detection of two radio sources identified as galaxies in the region observed by NIKA. The first source, hereafter RS1, is located in the southwest region of PSZ1 G045.85+57.71 at about 40 arcsec from the X-ray center. The second source, named RS2 in the following, is located at about 2 arcmin toward the north of the X-ray center. The fluxes of RS1 and RS2 are only significant at $1.4 \mathrm{GHz}$ and their values are reported in Table 2. We follow the method detailed in Adam et al. (2016) and model the radio source spectral energy distributions (SED) by a power law, $F_{v}=F_{1 \mathrm{GHz}}\left(\frac{v}{1 \mathrm{GHz}}\right)^{\alpha_{\text {radio }}}$, to estimate their expected fluxes in the NIKA bandpasses. As there is no other measurement of RS1 and RS2 fluxes referenced in other catalogs at different frequencies, we only constrain the $F_{1 \mathrm{GHz}}$ parameter and let the spectral index as a random variable following a Gaussian pdf centered on -0.7 and with a standard deviation of 0.2 , which is typical of radio galaxies (see Witzel 1979). The SEDs are thus simulated by computing Monte Carlo realizations of the radio source fluxes and spectral index within their error bars. The generated SEDs are then integrated within the NIKA bandpasses to predict the expected flux at 150 and $260 \mathrm{GHz}$. The results are reported in Table 3. Given the mean rms noise at the identified radio source locations we therefore conclude that their contamination at the NIKA frequencies is negligible.

We also consider the Herschel SPIRE (Griffin et al. 2010) data to identify submillimeter point sources and compute their expected fluxes at $150 \mathrm{GHz}$. Eleven sources are found in the region observed by NIKA thanks to the SPIRE $250 \mu \mathrm{m}$ cata$\log$. The corresponding sources in the other channels $(350 \mu \mathrm{m}$ and $500 \mu \mathrm{m})$ are inferred from their respective positions in the $250 \mu \mathrm{m}$ map. Following the procedure detailed in Adam et al. (2016), the fluxes of the sources are measured in the three SPIRE channels by fitting Gaussian functions at the source positions with a fixed FWHM given by the corresponding Herschel resolution in each channel $(35.2,23.9,17.6$ arcsec at 500, 350, and $250 \mu \mathrm{m}$ respectively). A local background is also fitted. Uncertainties on the flux of the sources are inferred by computing the dispersion of fluxes estimated by fitting the same Gaussian functions at random positions, where the noise is homogeneous. The $260 \mathrm{GHz}$ NIKA map was also used to constrain the SED slope at low frequency for each source. The computed submillimeter point source fluxes are presented in Table 4. The estimated fluxes corresponding to identified sources are compatible with the values reported in the Herschel catalog. A modified blackbody spectrum

$F_{v}=A_{0}\left(\frac{v}{v_{0}}\right)^{\beta_{\text {dust }}} B_{v}\left(T_{\text {dust }}\right)$

was used to model the SED of the identified submillimeter point sources from their estimated fluxes. In this model, $A_{0}$ is a normalization, $v_{0}$ a reference frequency, $\beta_{\text {dust }}$ the dust spectral index, and $T_{\text {dust }}$ the dust temperature. A Markov chain Monte Carlo 
Table 3. Best-fit parameters and extrapolation of the fluxes in the NIKA bands of the radio sources in the $4.4 \times 4.4$ arcmin $^{2}$ field around PSZ1 G045.85+57.71.

\begin{tabular}{ccccccccc}
\hline \hline Source & $\begin{array}{c}\text { RA offset } \\
{[\operatorname{arcsec}]}\end{array}$ & $\begin{array}{c}\text { Dec offset } \\
{[\operatorname{arcsec}]}\end{array}$ & $\begin{array}{c}F_{1 \mathrm{GHz}} \\
{[\mathrm{mJy}]}\end{array}$ & $\alpha_{\text {radio }}$ & $\begin{array}{c}F_{150 \mathrm{GHz}} \\
{[\mathrm{mJy}]}\end{array}$ & $\begin{array}{c}F_{260 \mathrm{GHz}} \\
{[\mathrm{mJy}]}\end{array}$ & $\begin{array}{c}\mathrm{rms}_{150 \mathrm{GHz}} \\
{[\mathrm{mJy}]}\end{array}$ & $\begin{array}{c}\mathrm{rms}_{260 \mathrm{GHz}} \\
{[\mathrm{mJy}]}\end{array}$ \\
\hline RS1 & -19.4 & -23.9 & $2.2 \pm 0.2$ & $-0.7 \pm 0.2$ & $0.11 \pm 0.11$ & $0.075 \pm 0.092$ & 0.36 & 1.9 \\
RS2 & 25.2 & 124.8 & $3.7 \pm 0.7$ & $-0.7 \pm 0.2$ & $0.18 \pm 0.19$ & $0.13 \pm 0.16$ & 0.42 & 2.2 \\
\hline
\end{tabular}

Notes. The mean rms noise on the flux of the identified point sources at their respective locations is also given at both NIKA frequencies. See text for details.

Table 4. Positions and fluxes of the 11 submillimeter sources identified in the $4.4 \times 4.4 \operatorname{arcmin}^{2}$ field around PSZ1 G045.85+57.71, measured by fitting Gaussian models to the Herschel maps at each wavelength as described in Sect. 3.2.

\begin{tabular}{|c|c|c|c|c|c|c|c|c|}
\hline Source & $250 \mu \mathrm{m}$ source position & $\begin{array}{c}250 \mu \mathrm{m} \\
{[\mathrm{mJy}]}\end{array}$ & $\begin{array}{c}350 \mu \mathrm{m} \\
{[\mathrm{mJy}]}\end{array}$ & $\begin{array}{c}500 \mu \mathrm{m} \\
{[\mathrm{mJy}]}\end{array}$ & $\begin{array}{c}1.15 \mathrm{~mm} \\
{[\mathrm{mJy}]}\end{array}$ & $\begin{array}{c}2.05 \mathrm{~mm} \\
\text { measured [mJy] }\end{array}$ & $\begin{array}{c}2.05 \mathrm{~mm} \\
\text { expected [mJy] }\end{array}$ & $\begin{array}{c}\mathrm{rms} \\
{[\mathrm{mJy}]}\end{array}$ \\
\hline SMG1 & $15: 18: 22.382,+29: 29: 03.42$ & $43.6 \pm 2.4$ & $51.4 \pm 3.4$ & $61.2 \pm 4.8$ & $1.9 \pm 2.2$ & $0.33 \pm 0.42$ & $0.27 \pm 0.09$ & 0.42 \\
\hline SMG2 & $15: 18: 16.978,+29: 27: 14.60$ & $36.7 \pm 2.3$ & $27.3 \pm 3.0$ & $13.6 \pm 3.5$ & $-2.1 \pm 1.9$ & $* *$ & $0.04 \pm 0.03$ & 0.36 \\
\hline SMG3 & $15: 18: 21.859,+29: 28: 30.33$ & $27.0 \pm 2.4$ & $-1.9 \pm 9.3$ & $2.1 \pm 9.8$ & $-1.3 \pm 2.1$ & $* *$ & $0.01 \pm 0.01$ & 0.38 \\
\hline SMG4 & $15: 18: 22.277,+29: 26: 49.87$ & $24.1 \pm 2.5$ & $9.5 \pm 9.3$ & $3.6 \pm 9.1$ & $-0.2 \pm 1.9$ & $-0.22 \pm 0.36$ & $0.07 \pm 0.05$ & 0.36 \\
\hline SMG5 & $15: 18: 25.577,+29: 28: 46.96$ & $19.4 \pm 2.2$ & $-4.1 \pm 9.5$ & $0.6 \pm 9.9$ & $-1.8 \pm 2.2$ & $0.27 \pm 0.43$ & $0.01 \pm 0.02$ & 0.43 \\
\hline SMG6 & $15: 18: 17.270,+29: 28: 33.72$ & $18.2 \pm 2.4$ & $14.6 \pm 3.4$ & $-0.6 \pm 9.3$ & $2.3 \pm 2.1$ & $0.08 \pm 0.38$ & $0.01 \pm 0.01$ & 0.39 \\
\hline SMG7 & $15: 18: 18.969,+29: 26: 20.75$ & $14.0 \pm 2.2$ & $-2.6 \pm 9.2$ & $9.0 \pm 10.2$ & $1.7 \pm 2.1$ & $0.26 \pm 0.39$ & $0.09 \pm 0.05$ & 0.39 \\
\hline SMG8 & $15: 18: 23.657,+29: 27: 36.72$ & $13.9 \pm 2.2$ & $-1.9 \pm 9.4$ & $-6.9 \pm 9.8$ & $-0.8 \pm 1.9$ & $0.21 \pm 0.36$ & $0.04 \pm 0.05$ & 0.36 \\
\hline SMG9 & $15: 18: 29.306,+29: 27: 03.83$ & $13.9 \pm 2.3$ & $17.3 \pm 3.1$ & $9.7 \pm 9.8$ & $3.2 \pm 3.3$ & $-0.29 \pm 0.48$ & $0.02 \pm 0.01$ & 0.48 \\
\hline SMG10 & $15: 18: 19.522,+29: 26: 44.27$ & $11.1 \pm 2.1$ & $9.7 \pm 3.1$ & $6.7 \pm 10.0$ & $1.1 \pm 1.9$ & $0.51 \pm 0.38$ & $0.04 \pm 0.02$ & 0.36 \\
\hline SMG11 & $15: 18: 18.545,+29: 27: 57.47$ & $8.1 \pm 2.2$ & $-4.0 \pm 9.0$ & $-3.1 \pm 9.7$ & $0.7 \pm 1.8$ & $* *$ & $0.01 \pm 0.01$ & 0.36 \\
\hline
\end{tabular}

Notes. The $260 \mathrm{GHz}$ NIKA map is also used to constrain each source SED at low frequency. Fluxes at 150 GHZ, which are not available because of the tSZ contamination are denoted by double stars **. The expected fluxes at $150 \mathrm{GHz}$ are computed by integrating the estimated SED in the NIKA bandpass. The final column corresponds to the NIKA $150 \mathrm{GHz}$ band rms noise at the source locations.

(MCMC) analysis was performed to compute the best-fit SED model for each source. The estimated SEDs are then integrated in the NIKA $150 \mathrm{GHz}$ bandpass to quantify the point source contamination at this frequency. The computed fluxes at $150 \mathrm{GHz}$ are reported in Table 4 and take the SPIRE color correction into account. These results show that the submillimeter point source contamination at $150 \mathrm{GHz}$ is one order of magnitude below the corresponding NIKA rms noise at this frequency. We therefore conclude that this contamination is negligible and do not consider either radio or submillimeter point sources in the ICM characterization developed in Sect. 5.

\subsection{XMM-Newton observations}

An X-ray follow-up of the Planck-discovered clusters has been undertaken since Spring 2010 (Planck Collaboration IX 2011). Thus, PSZ1 G045.85+57.71 has been observed for $\sim 24 \mathrm{ks}$ by the EPIC instruments during XMM-Newton revolution 2303 (2012 July 6). The basic data reduction (i.e., production of cleaned and calibrated event files, vignetting correction, point source removal, and the production of associated background data sets) followed the procedures described in Adam et al. (2016, and references therein). About $15 \mathrm{ks}$ of exposure time remained after the data cleaning.

The X-ray image shown in Fig. 2, combining the data from all three EPIC detectors, was produced as described in
Böhringer et al. (2010). Here the background subtraction, undertaken for each detector separately, was obtained from a model fit to an image with all sources (including the cluster) excised. The model, consisting of smoothly varying vignetted and unvignetted components, was normalized to the surface brightness in the outer cluster-free regions of the image and was then subtracted to the data.

\subsection{Multiprobe combined map of PSZ1 G045.85+57.71}

A combined map of observations of PSZ1 G045.85+57.71 with various probes is shown in Fig. 4 as a conclusion of this section. The identified point source positions are shown by 10 arcsec radii circles in cyan and yellow for the radio and submillimeter sources, respectively. The Hubble Space Telescope (HST) observed PSZ1 G045.85+57.71 on June 2015 with an exposure time of $1200 \mathrm{~s}$ (Ebeling 2014). The galaxy distribution (shown in green on the figure) identified by the HST using the $F 814 \mathrm{~W}$ filter follows an elliptical structure that is consistent with the ICM morphology measured by XMM-Newton and NIKA in red and blue, respectively. Although, the SZ and X-ray peak position are well aligned on the map, we cannot conclude on the cluster relaxation state because the NIKA S/N at $R_{500}$ is not high enough. However, the XMM-Newton observations along with the galaxy distribution reveal that this cluster has a significant elliptical morphology with a projected major axis oriented from the 

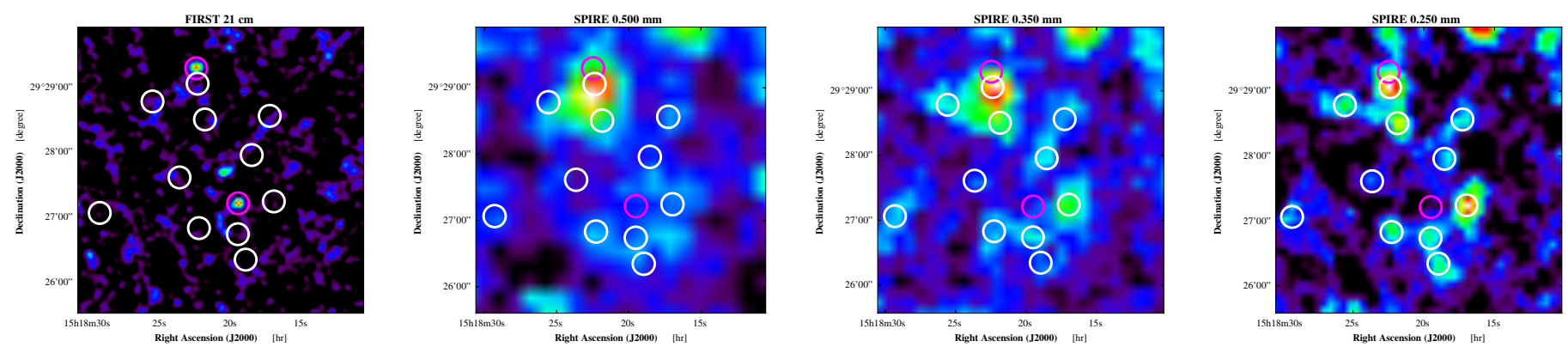

Fig. 3. Multiwavelength data set of PSZ1 G045.85+57.71 sky region (the displayed region is 4.4 arcmin wide). The considered instrument is indicated on the top of each map. The maps are smoothed and their range is adapted for visualization purposes. The 10 arcsec radius circles show the point source locations, in magenta for radio sources (Table 2) and in white for submillimeter sources (Table 4).

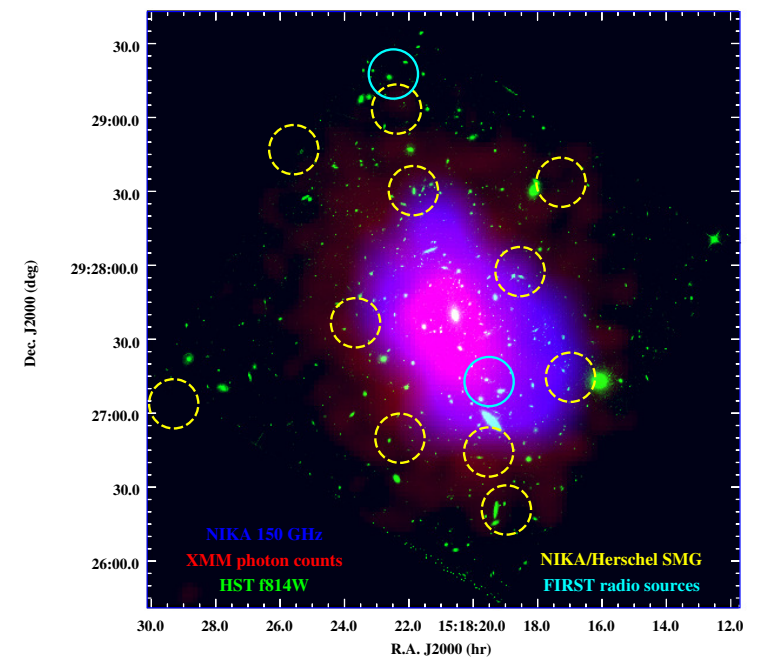

Fig. 4. Multiprobe combined map of PSZ1 G045.85+57.71. Blue: NIKA tSZ surface brightness map giving an estimate of the ICM pressure distribution. Red: XMM-Newton X-ray photon count map tracing the electronic density squared. Green: Hubble Space Telescope image of the cluster using the $F 814 \mathrm{~W}$ filter and showing the cluster galaxy locations. The solid cyan and dashed yellow circles give respectively the radio and submillimeter point sources identified in the field of view.

southwest of the X-ray center to the northeast. The next section describes how the PSZ1 G045.85+57.71 ICM is modeled in the following multiprobe analysis given the morphology constraints that we can derive from the NIKA tSZ surface brightness map.

\section{Modelization of the ICM}

\subsection{Parametric modeling}

The combination of NIKA and X-ray data, such as the $X M M-N e w t o n$ data, can bring new insights into the ICM thermodynamics reconstruction. Indeed, the electronic density within the ICM is low enough to consider it as an ideal gas for which the temperature is simply given by the ratio between the NIKA estimated pressure and the XMM-Newton estimated density at each point of the ICM. This method allows us to constrain the temperature profile of a galaxy cluster with almost no spectroscopic information. Indeed, the mean ICM temperature that is needed to deproject the cluster density profile can be estimated with only few spectroscopic data, whereas a temperature profile deprojection from spectroscopy measurements is time consuming at high redshift.
In the context of spherical cluster symmetry, we can model the ICM by the standard pressure and density models used in previous studies. The radial distribution of the cluster electronic pressure is modeled by a generalized Navarro-FrenkWhite (gNFW) profile (Nagai et al. 2007b), given by

$P_{\mathrm{e}}(r)=\frac{P_{0}}{\left(\frac{r}{r_{\mathrm{p}}}\right)^{c}\left(1+\left(\frac{r}{r_{\mathrm{p}}}\right)^{a}\right)^{\frac{b-c}{a}}}$,

where $P_{0}$ is a normalization constant, $r_{\mathrm{p}}$ is a characteristic radius, and $a, b$, and $c$ give the slope of the profile at intermediate, large, and small radii, respectively. The electronic density was modeled by a simplified Vikhlinin model (SVM) (Vikhlinin et al. 2006) given by

$n_{\mathrm{e}}(r)=n_{\mathrm{e} 0}\left[1+\left(\frac{r}{r_{\mathrm{c}}}\right)^{2}\right]^{-3 \beta / 2}\left[1+\left(\frac{r}{r_{\mathrm{s}}}\right)^{\gamma}\right]^{-\epsilon / 2 \gamma}$,

where $n_{\mathrm{e} 0}$ is the central density, $r_{\mathrm{c}}$ is the core radius, and $r_{\mathrm{s}}$ the transition radius at which an additional steepening in the profile occurs. The $\beta$ parameter gives the inner profile slope and $\epsilon$ the outer profile slope. The $\gamma$ parameter describes the width of the transition in the profile. In the following, we fix the $\gamma$ value at 3 since it provides a good fit to all clusters considered in the analysis of Vikhlinin (2006).

Models for both temperature and entropy profiles are naturally deduced from pressure and density if we consider the ICM as an ideal gas, $k_{\mathrm{B}} T_{\mathrm{e}}(r)=\frac{P_{\mathrm{e}}(r)}{n_{\mathrm{e}}(r)}$ and $K(r)=\frac{P_{\mathrm{e}}(r)}{n_{\mathrm{e}}(r)^{5 / 3}}$, where $k_{\mathrm{B}}$ is the Boltzmann constant. Assuming hydrostatic equilibrium, the total mass enclosed within the radius $r$ is then given by

$M_{\mathrm{HSE}}(r)=-\frac{r^{2}}{\mu_{\mathrm{gas}} m_{\mathrm{p}} n_{\mathrm{e}}(r) G} \frac{\mathrm{d} P_{\mathrm{e}}(r)}{\mathrm{d} r}$,

where $\mu_{\text {gas }}=0.61$ is the mean molecular weight of the gas, $m_{\mathrm{p}}$ the proton mass, and $G$ the Newton constant.

\subsection{Cluster mass estimation from a MCMC analysis}

In order to estimate the mass of the cluster, one must first evaluate both the density and pressure profiles describing the ICM. This ICM characterization method has been presented in detail in (Adam et al. 2015) and we only explain the key points of the analysis in this section. We use the information contained in the $150 \mathrm{GHz}$ tSZ surface brightness map to constrain the gNFW profile parameters from a MCMC sampling of the full parameter space. The main advantages of this approach are that we can use all the information contained in the map 
to constrain the parameters of interest, marginalize over nuisance parameters, and identify parameter correlations during the MCMC sampling. The inner slope of the pressure profile cannot be constrained at the considered cluster redshift because of the NIKA beam dilution. Therefore, all the gNFW parameters are kept free except for $c$, which is fixed to the value estimated by Planck Collaboration Int. V (2013) at $c=0.31$. At each step of the procedure, a set of parameters is generated and defines the corresponding pressure radial profile. The latter is integrated along the line of sight to compute a Compton parameter angular profile from which we derive a tSZ surface brightness map model $M_{\text {model }}$ at $150 \mathrm{GHz}$ and an integrated Compton parameter $Y_{\text {tot }}^{\text {model }}$ evaluated up to $5 R_{500}$. The relativistic corrections in the tSZ spectrum (Eq. (3)) are computed from the temperature estimate given by the ratio between the cluster pressure profile and its density profile. Both $M_{\text {model }}$ and $Y_{\text {tot }}^{\text {model }}$ are then compared to the observed $150 \mathrm{GHz}$ tSZ surface brightness map $\left(M_{\text {data }}\right)$ and integrated Compton parameter $\left(Y_{\text {tot }}^{\text {data }}\right)$ using the following Gaussian likelihood model:

$$
\begin{aligned}
-2 \ln \mathcal{L}= & \chi_{\text {SZ map }}^{2}+\chi_{Y_{\text {tot }}^{2}}^{2} \\
= & \sum_{i=1}^{N_{\text {pixels }}}\left[\left(M_{\text {data }}-M_{\text {model }}\right)^{T} C_{\text {NIKA }}^{-1}\left(M_{\text {data }}-M_{\text {model }}\right)\right]_{i} \\
& +\left(\frac{Y_{\text {tot }}^{\text {data }}-Y_{\text {tot }}^{\text {model }}}{\sigma_{\text {data }}}\right)^{2} .
\end{aligned}
$$

The MCMC sampling procedure also marginalizes over nuisance parameters such as the zero level of the NIKA map and the calibration coefficient uncertainty. The sampling stops when the convergence criteria given by Gelman \& Rubin (1992) is reached for all the fitted parameters. The final likelihood function marginalized distributions are eventually given by the remaining chain points after the burn-in cutoff, which discards the first $10 \%$ of each chain. These distributions are then used to compute the gNFW parameter constraints that define the best ICM pressure profile. Both density and pressure profiles are then used to compute a mass profile using Eq. (7) from which we can derive the cluster total mass $M_{500}$.

\subsection{Impact of the departure from sphericity on the ICM thermodynamic reconstruction}

A significant amount of disturbed clusters that are characterized, for instance, by the presence of substructures in the ICM, unvirialized ICM, or merging events, are identified at high redshift by high angular resolution observations.

In this context, describing the ICM by a spherical model may add dispersion and bias on ICM thermodynamic constrains and galaxy cluster mass estimations. In particular, this is the case if the intrinsic deviation from sphericity is significant, given the residual noise properties measured on the map.

This section describes the morphology analysis made on both XMM-Newton X-ray photon count map and NIKA tSZ surface brightness map at $150 \mathrm{GHz}$ to check the possibility to recover the cluster ellipticity in the individual maps. We then describe the analysis made on simulated tSZ surface brightness maps at $150 \mathrm{GHz}$ to study whether a spherical model is appropriate to derive PSZ1 G045.85+57.71 ICM thermodynamic properties from the NIKA and XMM-Newton observations.

\subsubsection{PSZ1 G045.85+57.71 ellipticity}

As shown in Fig. 2 (right panel), the XMM-Newton observations of PSZ1 G045.85+57.71 reveals a significant elliptical morphology of the ICM with a projected major axis oriented from the southwest of the X-ray center to the northeast. As the information along the line of sight is lost, we only constrain the length scales of this cluster in the plane of the sky. The ellipticity, defined by $\epsilon=1-\frac{b}{a}$, where $a$ and $b$ are the major and minor axes, respectively, of the considered ellipse, and the orientation of the major axis is estimated by fitting ellipses on iso-number count contours of the XMM-Newton photon count map. Their respective ellipticity and orientation were computed and show that PSZ1 G045.85+57.71 has a mean ellipticity of $\epsilon_{\mathrm{XMM}}=0.33 \pm 0.01$ and a major axis oriented with an angle $\theta_{\mathrm{XMM}}^{\mathrm{maj}}=(70 \pm 2)^{\circ}$ with respect to the RA axis in the clockwise direction. The uncertainties on both estimations are statistical only.

As the NIKA rms noise is fairly constant in the cluster region, the same analysis can been carried out on the NIKA tSZ surface brightness map using constant $\mathrm{S} / \mathrm{N}$ contours from 3.5 to 6.5 with 0.5 steps to fit the ellipses. This analysis shows a much larger dispersion on the estimated ellipticity and major-axis orientation with $\epsilon_{\mathrm{NIKA}}=0.4 \pm 0.1$ and $\theta_{\mathrm{NIKA}}^{\mathrm{maj}}=(44 \pm 8)^{\circ}$. The given error bars are statistical only and do not take the correlated noise on the map into account. Indeed, the residual correlated noise on the NIKA map can induce noise structures with angular scales larger than the NIKA beam at $150 \mathrm{GHz}$ that may distort the intrinsic ICM projected morphology. It is therefore important to characterize the bias induced by the spherical cluster assumption on the ICM thermodynamic reconstruction. Such a hypothesis will be adapted if the induced bias is negligible with respect to the uncertainty on the estimated constraints due to the residual noise on the NIKA map.

\subsubsection{Compatibility between NIKA SZ observations and the spherical cluster assumption}

Although the ellipticity observed on the NIKA map may be due to residual noise, PSZ1 G045.85+57.71 seems to be intrinsically elliptical as shown by the XMM-Newton map analysis (see Sect. 4.3.1). Therefore, to see if the spherical model assumption induces a significant bias on the final ICM characterization we choose the cluster total mass estimation as a quantitative indicator of the induced bias.

Knowing the cluster projected ellipticity and major-axis orientation from the XMM-Newton observations, we derive a tSZ surface brightness map from a simulated ellipsoidal cluster presenting similar projected morphological properties. The adopted model is a gNFW pressure profile with a modified radius defined as

$$
r=\sqrt{\left(\frac{x \cos (\theta)-y \sin (\theta)}{a}\right)^{2}+\left(\frac{x \sin (\theta)+y \cos (\theta)}{b}\right)^{2}+\left(\frac{z}{c}\right)^{2}}
$$

where $(a, b, c)$ define the axis ratios of the triaxial ICM and $\theta$ is the angle between the major axis and the line of sight. If the $\theta$ angle is different from $90^{\circ}$, the major-axis length has to be increased by a factor $1 / \sin (\theta)$ to keep the projected ellipticity unchanged. In the following, we consider the ideal case where the projected cluster ellipticity is equal to its intrinsic one (i.e., for a $\theta$ angle of $90^{\circ}$ ). The integration of this pressure model along the line of sight gives us a simulated tSZ surface brightness map 


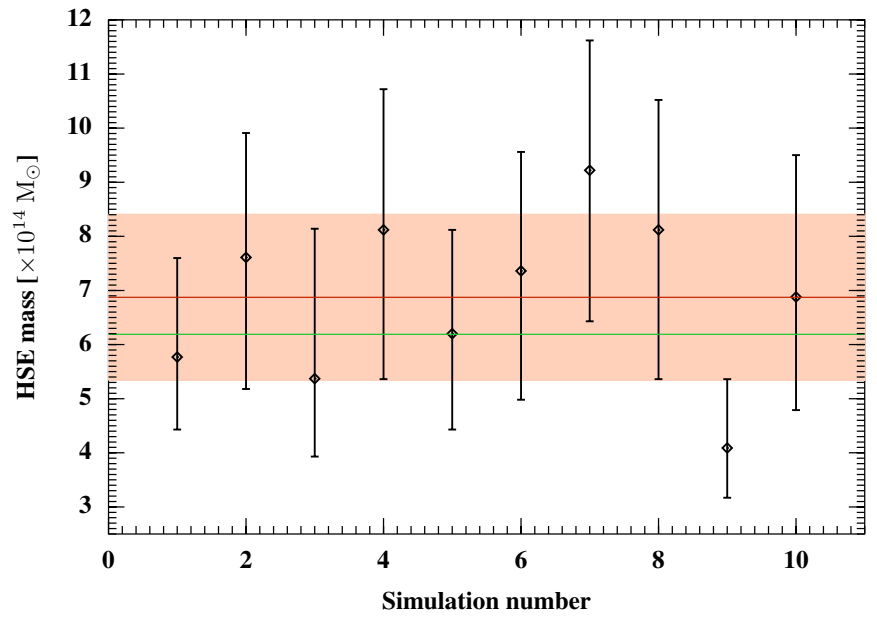

Fig. 5. Mass estimates (black diamonds) for the simulated triaxial clusters with their major axis orthogonal to the line of sight. The red line indicates the mean of the recovered distribution and the salmon region is its dispersion. The input mass given by the gNFW and SVM models considered for the simulation is represented by the green line.

model of an elliptical cluster. The tSZ surface brightness simulated maps are computed by adding residual noise to the modeled tSZ signal using the noise power spectrum derived from the NIKA null maps. The gNFW model parameters are adjusted so that the tSZ peak significance matches the one that we observe on the NIKA $150 \mathrm{GHz}$ map of PSZ1 G045.85+57.71.

We take the best-fit SVM model parameters of the PSZ1 G045.85+57.71 XMM-Newton data to model the simulated cluster density distribution (see Sect. 5.1) and use Eq. (9) for the modified radius definition to get an elliptical density distribution. The total mass of the simulated ellipsoidal cluster is estimated with the input pressure and density models in Eq. (7).

The simulated maps are then used to estimate the cluster total mass using a spherical model as described in Sect. 4.2. The estimated total masses of all Monte Carlo realizations are reported in Fig. 5. The input mass is shown as a green line while the salmon region indicates the $1 \sigma$ dispersion of the reconstructed mass distribution.

The reconstructed masses are consistent with the input model mass. We therefore conclude that the mass estimation given by a spherical model is not significantly biased by the cluster ellipticity if the cluster major axis is orthogonal to the line of sight for this residual noise level. The bias induced by the spherical model assumption on the reconstructed ICM thermodynamic properties is therefore negligible compared to the dispersion caused by the residual noise on the NIKA tSZ surface brightness map at $150 \mathrm{GHz}$.

We note however that if the cluster projected ellipticity is significantly different from its intrinsic one, the input gNFW parameter value has to be changed to get a tSZ surface brightness angular profile similar to the observed one. There is in particular a degeneracy between the $r_{\mathrm{p}}$ parameter value, the intrinsic cluster ellipticity, and the orientation of the major axis with respect to the line of sight. The reconstructed masses could therefore tend to be significantly biased because of projection effects (see for example Gavazzi 2005). We choose to develop this discussion in a forthcoming paper dedicated to simulation.

\section{Radial thermodynamical reconstruction}

Based on the results presented in the previous section, we assume the spherical symmetry of PSZ1 G045.85+57.71 in the following ICM characterization.

This section presents the different methods that have been used to deproject the radial pressure profile of PSZ1 G045.85+57.71. We first describe how the XMM-Newton data can be used to recover the electronic density and the gas temperature profiles of PSZ1 G045.85+57.71. This approach strongly depends on the spectroscopic temperature reconstruction, which is challenging at high redshift because of the cosmological dimming of the X-ray flux. Thus, spectroscopic temperature reconstruction at high redshift requires large integration time to recover the X-ray photon energy spectrum.

We then use the procedure described in Sect. 4.2 to estimate the best-fit gNFW pressure profile from the NIKA tSZ surface brightness map at $150 \mathrm{GHz}$ and the Planck/AMI combination of the integrated Compton parameter. This approach gives an estimation of the cluster radial pressure distribution without using spectroscopic information. However, it relies on a specific parametric model, which limits the use of the estimated pressure profile for future studies based on different ICM models.

We therefore choose to extract the pressure profile of PSZ1 G045.85+57.71 using a non-parametric spherical model to deproject the NIKA data in the MCMC analysis. Furthermore, instead of considering the Planck integrated Compton parameter to constrain the outer slope of the profile we use simultaneously the NIKA tSZ surface brightness map at $150 \mathrm{GHz}$ and the Planck MILCA map of the cluster Compton parameter in the MCMC. The whole ICM thermodynamics is then derived by combining the constrained pressure profile and the XMM-Newton density profile depending only weakly on X-ray spectroscopy.

\subsection{X-ray radial thermodynamic profiles}

Gas density profiles, $n_{\mathrm{e}}(r)$, produced from the [0.3-2] keV surface brightness profiles extracted directly from the event files centered on the emission peak, are obtained using the regularized deprojection and PSF-correction procedure described in Croston et al. (2006). Given the strongly constrained electronic density profile from the XMM-Newton data (shown in red on the left panel of Fig. 6), we choose to fit a SVM model on this profile independently and use it in the following multiprobe analysis. The SVM parameters are constrained by minimizing the $\chi^{2}$ on the deprojected density profile. The best-fit density profile is shown in blue in the figure and perfectly describes the $X M M-N e w t o n$ measurements. The reduced $\chi^{2}$ value is estimated at 1.4 .

The projected temperature profile was extracted in logarithmically spaced annuli as detailed in Pratt et al. (2010). The deprojected radial temperature profile, $T(r)$ (shown in red on the right panel of Fig. 6), was then obtained by convolving a parameteric model with a response matrix that simultaneously takes into account projection and PSF redistribution, projecting this model, and then fitting it to the projected annular profile. The projection procedure took the bias introduced by fitting isothermal models to multitemperature plasma into account (Mazzotta et al. 2004; Vikhlinin 2006). The computed temperature profile shows a typical cool-core shape, with a central temperature lower than $\sim 4 \mathrm{keV}$ and a peak of $\sim 8 \mathrm{keV}$ at about $200 \mathrm{kpc}$ away from the center.

The gas entropy $K(r)$ and pressure $P(r)$ distributions were obtained directly from the $3 \mathrm{D}$ density and temperature profiles 

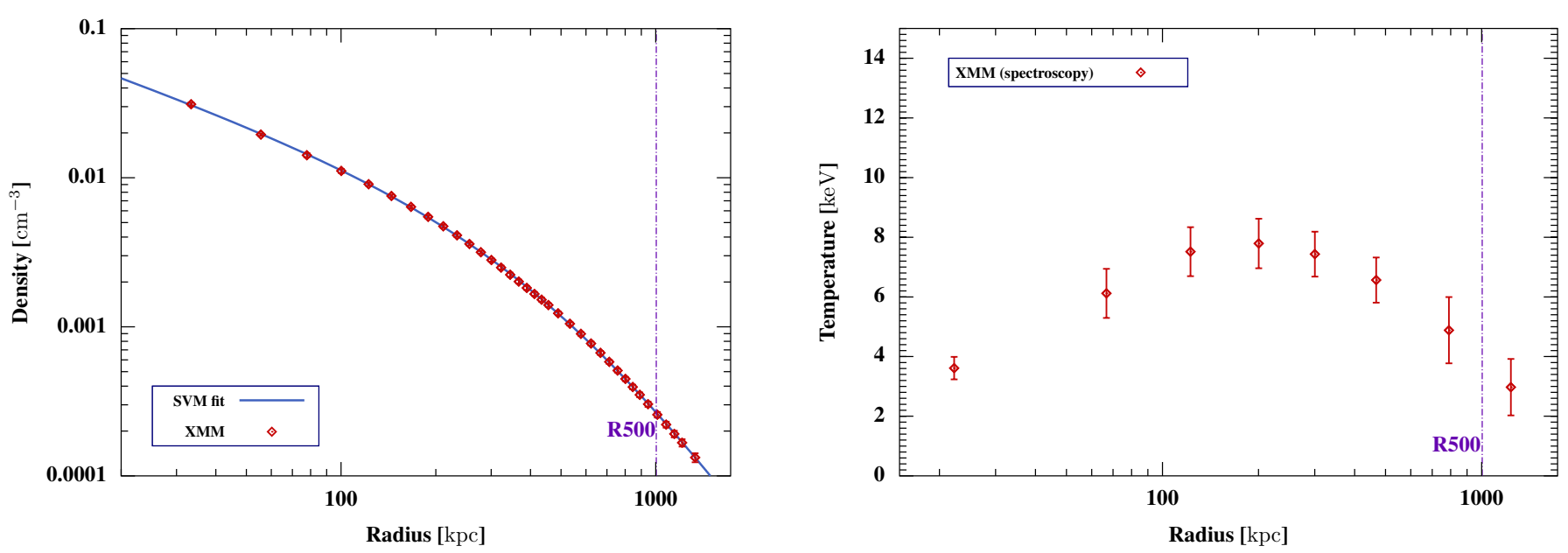

Fig. 6. Left: deprojected electronic density profile derived from the XMM-Newton data (red dots). The best-fit SVM model (Vikhlinin et al. 2006) is given by the blue line. Right: temperature profile derived from the XMM-Newton spectroscopy data. The characteristic radius measured from $X M M$-Newton data, $R_{500}=1013 \pm 13 \mathrm{kpc}$, is represented as a vertical dashed magenta line in both figures.

described above. The X-ray mass profile, $M_{\mathrm{HSE}}(r)$, derived assuming a spherical gas distribution in hydrostatic equilibrium, was calculated using the Monte Carlo procedure described in Démoclès et al. (2010, and references therein). The high quality of XMM-Newton spectroscopic data enables us to derive tight constraints on the whole ICM thermodynamics of PSZ1 G045.85+57.71 especially in the cluster core regions. However, such observations are becoming particularly challenging as cluster observations goes toward high redshift.

\subsection{MCMC analysis of the $150 \mathrm{GHz}$ NIKA map with a parametric model}

The entire thermodynamic properties of PSZ1 G045.85+57.71 can also be derived without spectroscopic information by using the cluster pressure profile constrained with the MCMC method described in Sect. 4.2 and the XMM-Newton deprojected density profile jointly. The $150 \mathrm{GHz}$ NIKA map is used to constrain both the inner and intermediate parts of the gNFW pressure profile. The Planck/AMI combined estimation of the cluster integrated Compton parameter $Y_{5 R 500}^{\text {Planck/AMI }}=(1.47 \pm 0.51) \times 10^{-3} \mathrm{arcmin}^{2}$ is used in the MCMC analysis to constrain the outer slope of the gNFW pressure profile.

The panel a of Fig. 7 shows the marginalized distributions (diagonal) and 2D correlations (off-diagonal) of the considered parameters. These distributions are used to compute the gNFW parameter constraints that define the best ICM pressure profile. As shown in Fig. 7, the degeneracy between the $r_{\mathrm{p}}$ and $b$ parameters is very strong. All the parameter degeneracies are taken into account when estimating the final pressure profile error bars. The panel $b$ of Fig. 7 shows that the NIKA+Planck/AMI pressure profile estimated with this method is compatible with the $X M M-N e w t o n$ constrained points within error bars.

The constraints on the pressure profile come almost exclusively from the NIKA and Planck/AMI data. However, we account for relativistic corrections in the tSZ spectrum using the radial temperature profile estimated by combining the deprojected pressure profile with the XMM-Newton constrained density profile. The overall effect on the final pressure profile is very small compared to the uncertainties coming from residual noise on the NIKA map. The Planck/AMI estimation of the integrated Compton parameter allows the MCMC procedure to avoid models that diverge at large scales, where NIKA is not sensitive and partially breaks the strong degeneracy between the zero level and the $r_{\mathrm{p}}$ parameter (see Fig. 7). Planck and NIKA are therefore highly complementary to constrain the pressure profile from small to large scales.

As shown in the panel b of Fig. 7, the NIKA estimated uncertainties increase in both the cluster core and its outskirts because of the analysis filtering, beam dilution, and the larger rms noise outside the NIKA FOV, respectively. The most constrained region of the cluster using this method lies therefore between projected angular scales from the X-ray center of about 0.5 and 2 arcmin, which correspond for this cluster redshift to distances from the X-ray center of 200 and $800 \mathrm{kpc}$, respectively.

\subsection{MCMC analysis based on a non-parametric model}

The previous MCMC analysis has been upgraded to fully constrain the ICM pressure distribution from the cluster core to its outskirts and to improve on the integrated Compton parameter estimation. In this section, we present the new model that constrains the ICM pressure distribution, the new likelihood function used in the MCMC procedure, and the results obtained with this method.

We use a non-parametric model to constrain the cluster pressure distribution in the framework of spherical symmetry to study potential deviations from the standard self-similar assumption (e.g., Basu et al. 2010). Instead of constraining the gNFW model parameters in the MCMC sampling, we constrain the values of the pressure at different distances from the X-ray center from the cluster core to its outskirts. The pressure between the constrained points is defined with a power law interpolation. We allow $P\left(r_{i}\right)$ and $P\left(r_{i+1}\right)$ to be the constrained pressure at the $r_{i}$ and $r_{i+1}$ radii from the $\mathrm{X}$-ray center, where the pressure at a radius $r \in\left[r_{i}, r_{i+1}\right]$ is defined as

$P(r)=P\left(r_{i}\right) \times 10^{\alpha} \quad$ with $\quad \alpha=\frac{\log _{10}\left(\frac{P\left(r_{i+1}\right)}{P\left(r_{i}\right)}\right) \times \log _{10}\left(\frac{r}{r_{i}}\right)}{\log _{10}\left(\frac{r_{i+1}}{r_{i}}\right)}$.

The pressure profile radial bins were defined with an increased sampling of the pressure profile in the region mainly constrained 
a)
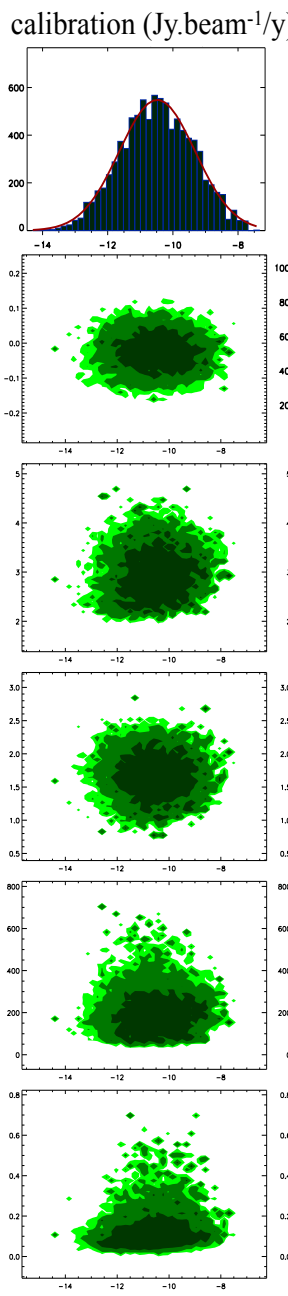

zero level (mJy/beam)
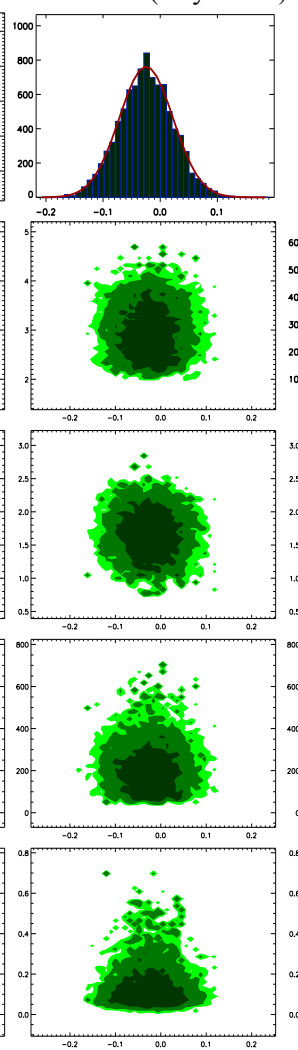

b)
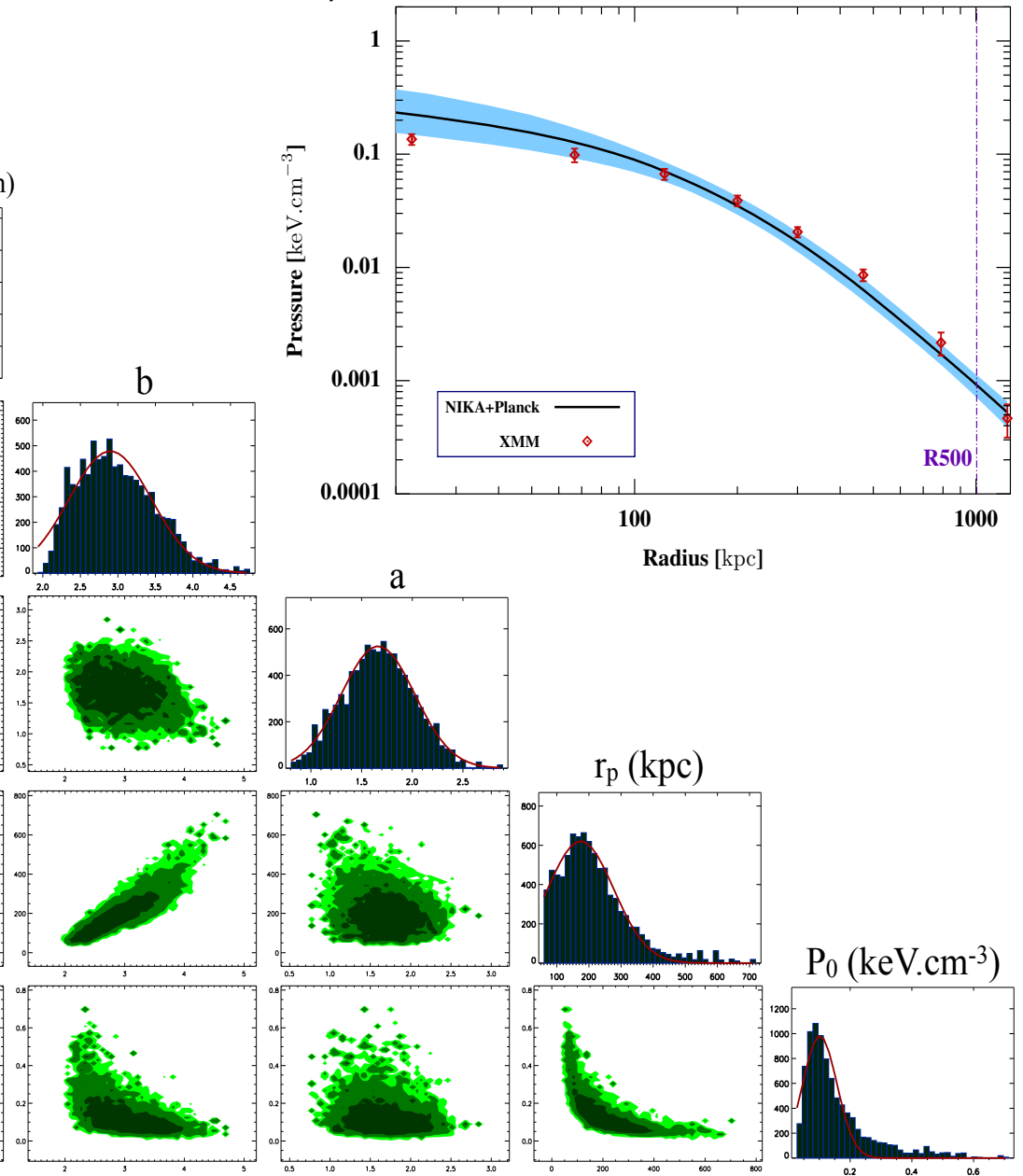

Fig. 7. Panel a): marginalized distributions (diagonal) and 2D correlations (off-diagonal) plots of the parameters of the assumed gNFW model. The MCMC procedure constrains the parameters of interest (from the bottom of the diagonal to the top): $P_{0}, r_{\mathrm{p}}, a$, and $b$ and marginalize over the map zero level and $y$-mJy/beam calibration coefficient. Panel b): maximum likelihood NIKA+Planck/AMI pressure profile (black), the 1-sigma dispersion (light blue), and XMM-Newton constrained pressure profile (red).

by the NIKA map (see Sect. 5.2). Eleven pressure profile radial bins are defined from $\sim 0.02 R_{500}$ to $\sim R_{500}$, which are mainly constrained by the NIKA tSZ surface brightness map, and 3 bins at 3,4 , and $\sim 5 R_{500}$, which are constrained by the Planck Compton parameter map.

Indeed, instead of using the Planck/AMI estimation of the integrated Compton parameter to partially break the degeneracy between the map zero level and the pressure profile characteristic radius, the Compton parameter map of PSZ1 G045.85+57.71 (see Fig. 2 left panel) that is obtained with MILCA (Planck Collaboration XXII 2016), $y_{\text {Planck, }}$, is used in combination with the NIKA map, $M_{\mathrm{NIKA}}$, to simultaneously constrain the cluster pressure profile at intermediate and large angular scales.

We simulated Planck noise maps using the variance per pixel and homogeneous noise power spectrum provided by Planck Collaboration XXII (2016). These simulations are used to compute the pixel-to-pixel noise covariance matrix in the considered region of the Planck MILCA $y$-map $C_{\text {Planck. }}$ At each step of the MCMC sampling, a pressure profile is defined using Eq. (10) and is used to derive a tSZ surface brightness map, $M_{\text {model }}$ at $150 \mathrm{GHz}$, and a Compton parameter map $y_{\text {model }}$. They are then respectively compared to the NIKA and Planck data via the following likelihood function:

$$
\begin{aligned}
-2 \ln \mathcal{L}= & \chi_{\text {NIKA }}^{2}+\chi_{\text {Planck }}^{2} \\
= & \sum_{i=1}^{N_{\text {pixels }}^{\text {NIKA }}}\left[\left(M_{\text {NIKA }}-M_{\text {model }}\right)^{T} C_{\text {NIKA }}^{-1}\left(M_{\text {NIKA }}-M_{\text {model }}\right)\right]_{i} \\
& +\sum_{j=1}^{N_{\text {piacls }}^{\text {Plack }}}\left[\left(y_{\text {Planck }}-y_{\text {model }}\right)^{T} C_{\text {Planck }}^{-1}\left(y_{\text {Planck }}-y_{\text {model }}\right)\right]_{j} .
\end{aligned}
$$

Uniform priors spanning from 0 to $2 \mathrm{keV} \mathrm{cm}^{-3}$ are used for each pressure bin. This MCMC procedure also marginalizes over the zero level of the NIKA map and the calibration coefficient uncertainty. The correlations between the constrained pressure points are taken into account as in Sect. 5.2 to estimate the error bars on the pressure profile.

We tested this method on simulations to check the pressure profile reconstruction. An input pressure distribution modeled as a gNFW profile was used to simulate tSZ surface brightness maps on which residual correlated noise was added using the 


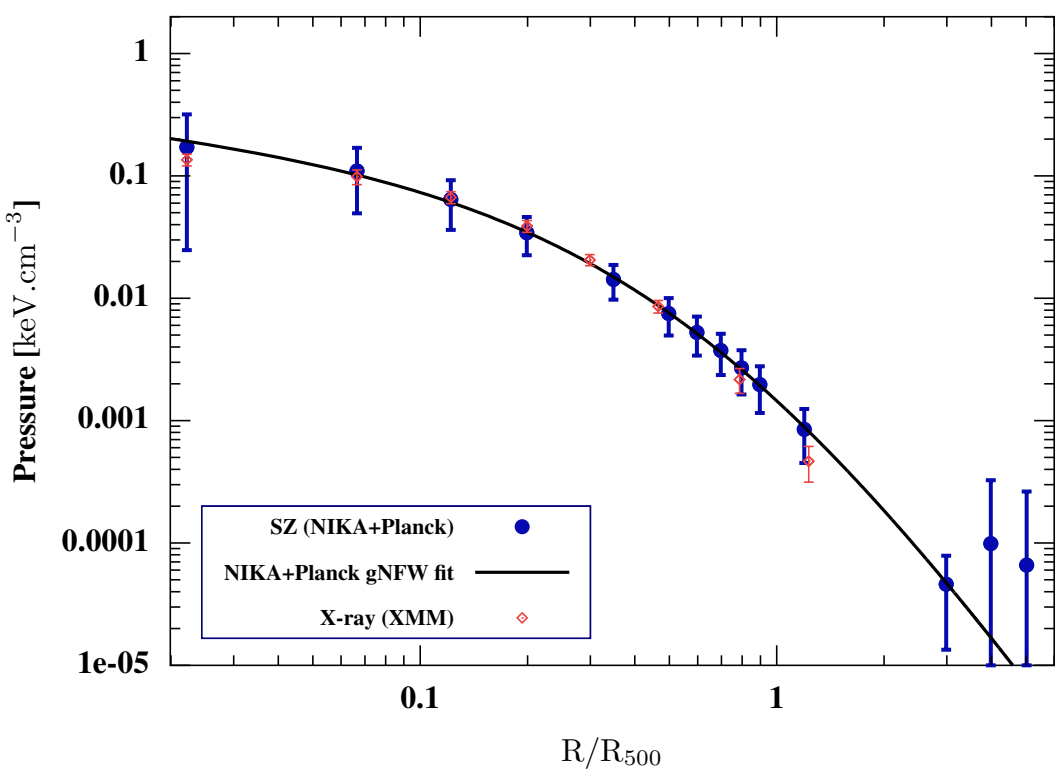

Fig. 8. Non-parametric pressure profile (blue) deprojected from the NIKA tSZ surface brightness map and the Planck Compton parameter map. The 3 radial bins at 3,4 , and $5 R_{500}$ are mostly constrained by the Planck data. A gNFW pressure profile model has been fitted on the NIKA+Planck deprojected pressure points (black solid line). XMM-Newton estimated pressure profile (red) based on the deprojected density profile and the temperature estimation from spectroscopic observations. The NIKA/Planck and XMM-Newton estimates are compatible within error bars. noise power spectrum derived from the NIKA null-maps. The constrained pressure points are always consistent with the input pressure profile.

The NIKA+Planck deprojected pressure profile of PSZ1 G045.85+57.71 is shown in Fig. 8 along with the XMM-Newton estimate in red. The pressure within the ICM is constrained from the cluster core to its outskirts without relying on X-ray spectroscopy at the intermediate redshift $z=0.61$. Such a non-parametric pressure profile deprojection is comparable with what has been achieved with the Planck satellite for low redshift $(z<0.2)$ galaxy clusters (see Planck Collaboration Int. V 2013).

The $1 \sigma$ error bars of the deprojected pressure points are larger than the error bars we get from the previous MCMC analysis because the pressure profile is only constrained by the data whereas a parametric model fitting induces additional constraints. In the context of the spherical cluster assumption, such a non-parametric pressure profile deprojection gives an estimate of the intrinsic ICM pressure distribution without model-induced bias.

Considering the Planck/AMI integrated Compton parameter in the MCMC analysis enables us to avoid models that diverge at large scales but does not takeall the information contained in the Planck Compton parameter map into account. Using the whole Planck Compton parameter map in the likelihood estimation allows us to constrain both the normalization of the pressure profile and the pressure distribution at large scales where NIKA is not sensitive. Therefore, the three pressure profile radial bins constrained by the Planck map at large radii give a strong constraint on the pressure profile slope in the cluster outskirts. This highlights the complementarity between large FOV experiments, albeit with low resolution, such as Planck and the NIKA instrument, which benefit from the IRAM $30 \mathrm{~m}$ telescope high resolution.

All the deprojected pressure values in Fig. 8 are compatible with the pressure profile derived with the previous MCMC analysis based on a gNFW modeling of the pressure distribution (see Sect. 5.2). The pressure profile of PSZ1 G045.85+57.71 is therefore well-described by a gNFW model. The agreement between NIKA/Planck and XMM-Newton estimates is good as detailed in the following section.
The maximum likelihood tSZ surface brightness map and Compton parameter map have been used to compute residual maps for both NIKA and Planck observations. The top and bottom panels of Fig. 9 show the raw data, maximum likelihood model, and residual maps for NIKA and Planck, respectively. Although residuals are seen in the southwest region of the NIKA map, the $\mathrm{S} / \mathrm{N}$ in both residual maps is always lower than 3, which therefore allows us to conclude that there are no significant substructures in PSZ1 G045.85+57.71 and that the NIKA $150 \mathrm{GHz}$ map of this cluster is well described by a projected spherical model, given the amount of residual correlated noise.

\subsection{Integrated Compton parameter estimation}

Both parametric and non-parametric deprojected pressure profiles are then used to estimate the cluster integrated Compton parameter $Y_{500}^{\text {param }}=4.23_{-0.62}^{+0.68} \times 10^{-4} \operatorname{arcmin}^{2}$ and $Y_{500}^{\text {non-param }}=5.61_{-0.59}^{+0.68} \times 10^{-4} \operatorname{arcmin}^{2}$, which are in agreement with the Planck estimation $Y_{500}=8.21_{-1.70}^{+1.73} \times 10^{-4} \mathrm{arcmin}^{2}$ (Planck Collaboration XXXII 2015). The best relative uncertainty is obtained with the non-parametric pressure profile deprojection because this method gives the most stringent constraints on the cluster pressure distribution from its core up to $5 R_{500}$. Thus, the relative uncertainty on the integrated Compton parameter tracing the total thermal energy within the ICM is improved by a factor 2 with respect to the Planck estimate because the pressure profile is much more constrained at each scale. Furthermore, the NIKA high angular resolution allows us to completely break the $\theta_{\mathrm{s}}-Y_{\text {tot }}$ degeneracy observed in both Planck and AMI observations (Perrott et al. 2015).

This result highlights the necessity of a high resolution tSZ follow-up of Planck-discovered clusters to better constrain the $Y-M$ scaling relation used for future cosmology studies. Indeed, a non-parametric joint analysis of both NIKA and Planck data leads to a deprojected pressure profile, which is constrained at every scale and is not affected by model-induced bias. This approach allows us to give a stringent constraint on the integrated Compton parameter used to calibrate the $Y-M$ scaling relation. The PSZ1 G045.85+57.71 integrated Compton parameter estimations derived from SZ observations are summarized in Table 5. 

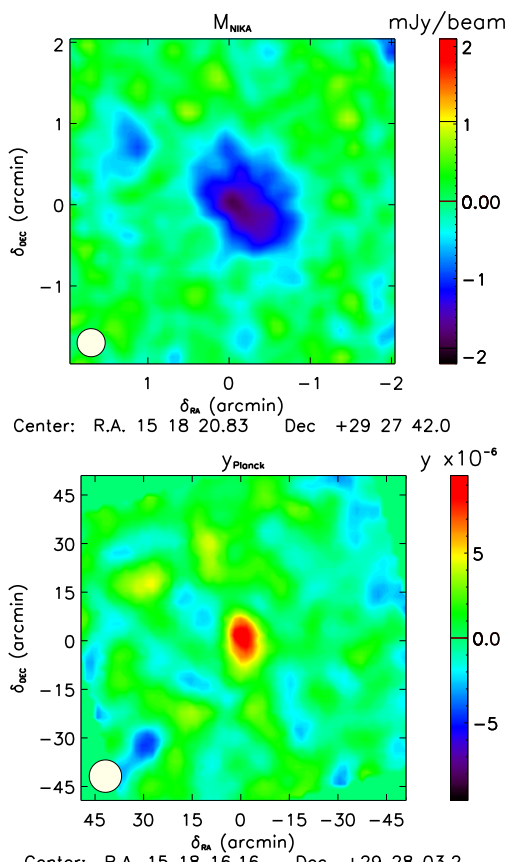
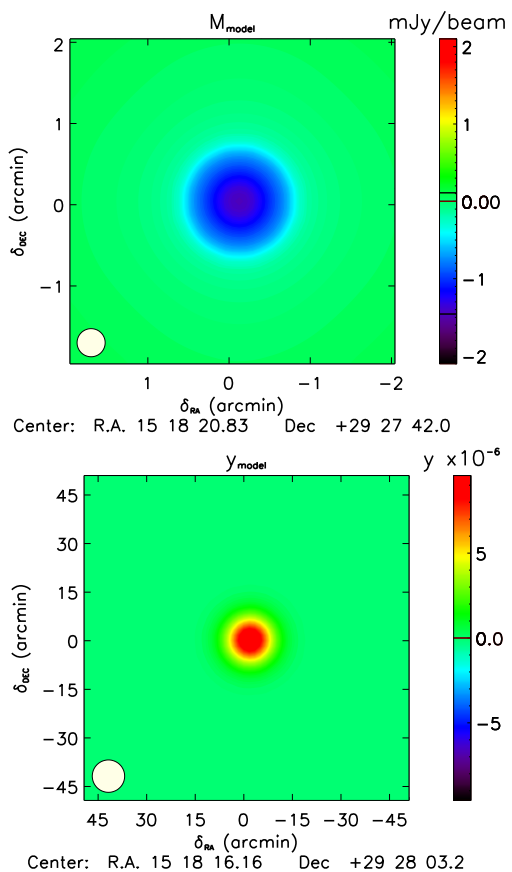
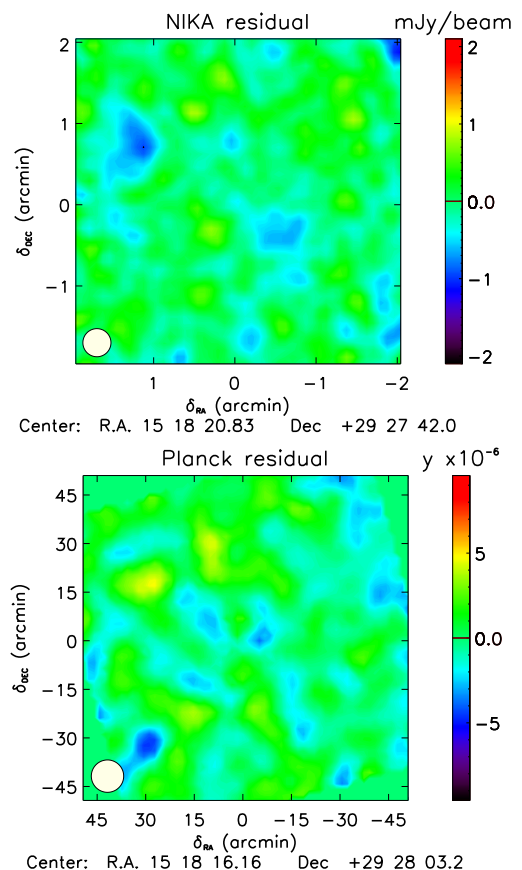

Fig. 9. NIKA tSZ surface brightness at $150 \mathrm{GHz}$, maximum likelihood tSZ map and residual (top row) and Planck Compton parameter map, maximum likelihood Compton parameter map and residual (bottom row) computed from a non-parametric model based MCMC analysis. The residual maps does not indicate any significant substructure since no $\mathrm{S} / \mathrm{N}$ over 3 is observed. The NIKA beam at $150 \mathrm{GHz}$ and the Planck effective beam of 10 arcmin FWHM are shown in the bottom left-hand corner of the top and bottom row maps, respectively.

Table 5. Estimations of PSZ1 G045.85+57.71 integrated Compton parameter $\left(Y_{500}\right)$ from the constraint derived by Planck (Planck Collaboration XXXII 2015), from the 2D model based MCMC analysis (see Sect. 4.2), and from the non-parametric model based MCMC analysis (see Sect. 5.3).

\begin{tabular}{ccc}
\hline \hline Data & Method & $Y_{500}\left(\operatorname{arcmin}^{2}\right)$ \\
\hline Planck & catalog & $8.21_{-1.70}^{+1.73} \times 10^{-4}$ \\
NIKA + Planck/AMI & parametric & $4.23_{-0.62}^{+0.68} \times 10^{-4}$ \\
NIKA + Planck map & non-parametric & $5.61_{-0.59}^{+0.68} \times 10^{-4}$ \\
\hline
\end{tabular}

\subsection{Thermodynamics of the cluster}

In this section, we use the complementarity between NIKA and $X M M-N e w t o n$ data sets to fully constrain the thermodynamics of PSZ1 G045.85+57.71. As both the cluster density and pressure profiles estimated from non-parametric deprojection methods are consistent with parametric models (see Figs. 6 and 8), we choose for convenience to combine the best-fit SVM model of the XMM-Newton density profile and the best-fit gNFW model of the NIKA/Planck pressure profile to constrain the whole ICM thermodynamics.

The maximum likelihood pressure values computed from the non-parametric analysis were therefore fitted by a gNFW model by taking into account the correlations between each pressure points. A reduced $\chi^{2}$ of 1.13 was computed for the fit. This emphasizes the good agreement between the NIKA deprojected pressure points and the standard gNFW model. The pressure profile constrained by the $150 \mathrm{GHz}$ NIKA map is shown in Fig. 10 (top left panel) in black together with the deprojected pressure points from the XMM-Newton analysis in red. All the $X M M-N e w t o n$ estimated pressure points are compatible with the NIKA constrained pressure profile within the $68 \%$ confidence level uncertainties shown in blue in Fig. 10. The XMM-Newton estimate of the pressure profile can only be inferred with spectroscopic information while tSZ observations directly probe the pressure distribution within the ICM. Comparing both estimated pressure profiles allows us then to bring strong constraints on the cluster pressure distribution as the two methods are completely independent.

The NIKA pressure profile estimate is compared with the universal pressure profile computed using the REXCESS representative sample of nearby clusters (Böhringer et al. 2007; Arnaud et al. 2010). The solid and dashed orange lines in Fig. 10 (top left panel) give the cool-core and morphologically disturbed subsample mean pressure profile, respectively. The normalization of the two profiles was computed using the XMM-Newton total mass estimation taking into account the mass dependence of the shape of the profile (Arnaud et al. 2010). As shown in the bottom part of Fig. 10 (top left panel), the cool-core and morphologically disturbed cluster universal profiles are both within the $2 \sigma$ error bars of the PSZ1 G045.85+57.71 pressure profile estimation. Therefore, the NIKA estimated profile alone does not bring significant information on the relaxation state of this cluster.

The cluster temperature, entropy, and mass profiles were computed by combining both the NIKA estimated pressure profile and the fitted SVM density profile as explained in Sect. 4.1.

The estimated temperature profile shown in Fig. 10 (top right panel) is compatible with that estimated from the XMM-Newton spectroscopic observations and its shape is consistent with that expected for a cool-core cluster. The core temperature goes down to $\sim 4 \mathrm{keV}$ and the maximum temperature of $\sim 7 \mathrm{keV}$ is reached at a distance of $\sim 200 \mathrm{kpc}$ from the X-ray center. The NIKA-XMM combined temperature profile (without spectroscopy) seems to be flatter in the cluster outskirts than that estimated by the XMM data alone (with spectroscopy). This could be an indication of 

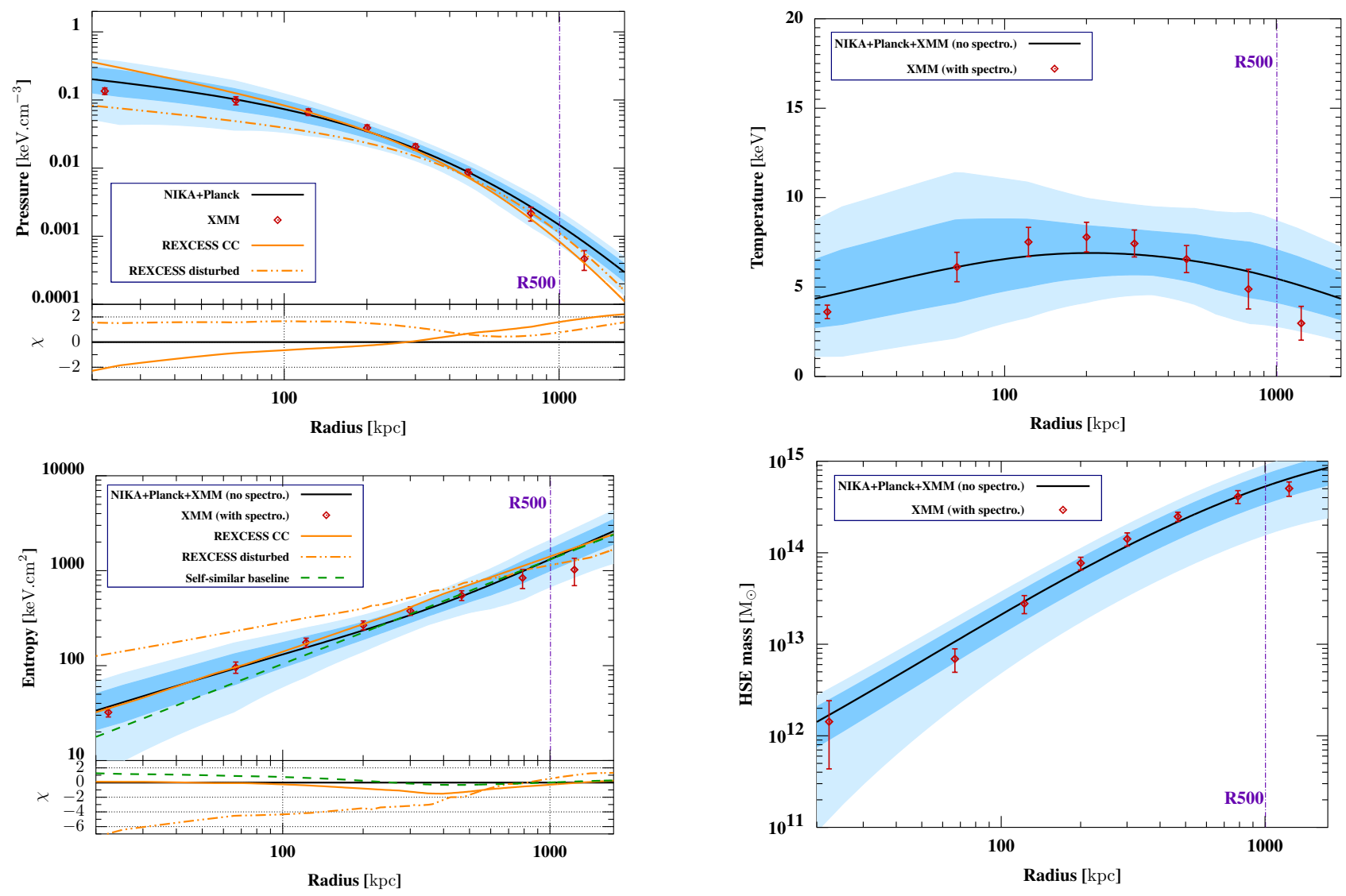

Fig. 10. MCMC constraints on the deprojected radial profiles of the pressure (top left), temperature (top right), entropy (bottom left), and hydrostatic mass (bottom right) based on the non-parametric method. The XMM-Newton only measurements are indicated with red dots. The dark and light blue regions show the $68 \%$ and $95 \%$ confidence limit on the NIKA/Planck estimated profiles, whose best fit is indicated by the black line. The pressure (Arnaud et al. 2010) and entropy (Pratt et al. 2010) mean profiles of both cool-core (orange solid line) and morphologically disturbed (orange dashed line) clusters based on a representative sample of nearby X-ray clusters is also shown. The weighted difference between these mean profiles and the NIKA/Planck estimated profile is shown in the bottom part of both pressure and entropy panels. For the entropy profile, the self-similar expectation computed from non-radiative simulations (Voit et al. 2005) is also represented as a green dashed line.

clumping in the cluster outskirts. However, this trend is not significant compared to the error bars.

The estimated entropy profile is shown in Fig. 10 (bottom left panel) along with the XMM-Newton results. As shown in Voit (2005), the entropy distribution in the ICM traces the thermodynamical history of the gas and is a good estimator of its relaxation state. A baseline entropy profile was computed by Voit et al. (2005) using numerical simulations without including hydrodynamical processes. The latter, converted from an overdensity of 200 to 500, takes the form of a power law scaled by a factor depending on the cluster mass and baryon mass fraction (Pratt et al. 2010),

$K(r)=1.42 K_{500}\left(R / R_{500}\right)^{1.1}$ with

$K_{500}=106 \mathrm{keV} \mathrm{cm}^{-2}\left(\frac{M_{500}}{10^{14} h_{70}^{-1} M_{\odot}}\right)^{2 / 3}\left(\frac{1}{f_{b}}\right)^{2 / 3} E(z)^{-2 / 3} h_{70}^{-4 / 3}$.

The corresponding self-similar baseline was computed for this cluster and is shown as a green dashed line in Fig. 10 (bottom left panel). The concordance between this baseline and the estimated entropy profile is very good especially outside the cluster core where the non-gravitational processes have less impact on the derived thermodynamic constraints. In order to have a better description of the entropy distribution in both the cluster core and its periphery, it can be modeled by a power law plus constant profile (Donahue et al. 2006),

$K(r)=K_{0}+K_{100}\left(\frac{r}{100 h_{70}^{-1} \mathrm{kpc}}\right)^{\alpha}$.

This model describes well the higher plateau and shallower slope observed on disturbed system entropy profiles. The NIKA-XMM combined entropy profile is compared with the mean entropy profiles estimated from the REXCESS representative subsamples of cool-core and morphologically disturbed clusters using the best-fit estimations of these model parameters (Pratt et al. 2010). The results are shown in Fig. 10 (bottom left panel) using a solid line and a dashed orange line for the cool-core and morphologically disturbed clusters, respectively. The bottom part of the figure shows the weighted difference between the NIKAXMM estimated entropy profile and the considered models. The mean entropy profile computed from the REXCESS representative subsample of cool-core clusters is in very good agreement with the NIKA-XMM estimated profile especially in the cluster core. The mean profile describing the morphologically disturbed cluster entropy distribution is however in strong tension with the observed profile. Indeed, the deviation from the NIKA-XMM estimated profile is higher than $3 \sigma$ from the X-ray center up to radial scales of $\sim 400 \mathrm{kpc}$. The estimated entropy profile allows 
us to conclude that PSZ1 G045.85+57.71 is a cool-core cluster, confirming the indications from the temperature profile. This emphasizes the complementarity between tSZ and X-ray observations to constrain the full thermodynamic state of a cluster.

The hydrostatic equilibrium hypothesis was assumed to derive the mass profile of PSZ1 G045.85+57.71 as described in Sect. 4.1. The estimated profile is shown in the bottom right panel of Fig. 10 along with the XMM-Newton constraints using only X-ray data. All the XMM-Newton estimated values are compatible with the NIKA-XMM combined profile within the $68 \%$ confidence limit. The estimated mass profile was then used to compute the cluster characteristic radius $R_{500}=1004_{-161}^{+202} \mathrm{kpc}$ and total mass within $R_{500}, M_{500}=\left(5.4_{-3.0}^{+2.6}\right) \times 10^{14} M_{\odot}$. We do not expect to obtain constraints as stringent as those derived from an X-ray based analysis because the reconstructed NIKA pressure profile shows a larger dispersion. Nevertheless, these results are compatible with the XMM-Newton estimations using spectroscopic observations: $R_{500}^{\mathrm{XMM}}=1013 \pm 13 \mathrm{kpc}$ and $M_{500}^{\mathrm{XMM}}=(5.78 \pm 0.21) \times 10^{14} M_{\odot}$ and show that $\mathrm{tSZ}$ observations are a good alternative to derive cluster thermodynamic properties even at high redshift, where accurate X-ray spectroscopy measurements require large integration time.

\section{Conclusions and perspectives}

The Planck tSZ-discovered cluster PSZ1 G045.85+57.71 has been observed simultaneously at 150 and $260 \mathrm{GHz}$ by the NIKA camera. A $4.35 \mathrm{~h}$ observation allowed a detailed mapping at 18.2 arcsec angular resolution of the $\mathrm{tSZ}$ signal at $150 \mathrm{GHz}$. The cluster was also observed in the X-ray band by the XMM-Newton satellite.

We performed the first non-parametric pressure profile deprojection from resolved tSZ observations of a Planckdiscovered cluster at an intermediate redshift $(z=0.61)$. The MCMC procedure, which was developed to deproject the cluster pressure profile, uses the NIKA tSZ surface brightness map and the Planck Compton parameter map jointly to constrain the cluster pressure distribution from its core up to $5 R_{500}$. The resulting pressure profile does not deviate significantly from the standard gNFW model.

The combination of both NIKA and Planck data brings strong constraints on the pressure profile slope at each scale, and allows a significant improvement in the relative uncertainty on the integrated Compton parameter value $Y_{500}$. The latter highlights the utility of high resolution tSZ follow-up of Planckdiscovered clusters to better constrain the $Y-M$ scaling relation used for cosmology studies based on cluster counts (Comis et al. 2016).

We further combined the NIKA+Planck deprojected non-parametric pressure profile with the deprojected electronic density profile obtained from XMM-Newton observations. This allowed us to obtain temperature and entropy profiles without recourse to X-ray spectroscopy and to undertake an hydrostatic mass analysis. The X-ray only (including spectroscopy) and the tSZ+X-ray (without spectroscopy) constraints are consistent within their uncertainties. This shows that high resolution tSZ observations, combined with X-ray snapshot imagery, are a competitive alternative to constrain cluster thermodynamics at high redshift, where X-ray spectroscopy requires large integration times to derive accurate temperature estimates. Comparison of the thermodynamic profiles to those obtained from the representative X-ray sample REXCESS (Böhringer et al. 2007; Arnaud et al. 2010; Pratt et al. 2010), in particular the radial distributions of temperature and entropy, indicates that PSZ1 G045.85+57.71 is a cool-core cluster. This result illustrates the complementarity between tSZ and X-ray data when only X-ray imaging observations are available.

The NIKA2 camera now installed at the focal plane of the IRAM 30-m telescope is currently undergoing commissioning. The number of detectors has been increased by a factor 10 with respect to the NIKA prototype to fully sample the telescope field of view of 6.5 arcmin. The NIKA2 tSZ Guaranteed Time Large Program (Mayet et al. 2016) is a follow-up of $50 \mathrm{SZ}$-discovered clusters with redshift up to $z=1$, selected from the Planck and ACT catalogs (Hasselfield et al. 2013; Planck Collaboration XXVII 2016). Following the work presented in this paper and in the previous NIKA studies (Adam et al. 2014, 2015, 2016), NIKA2 is expected to provide reliable tSZ detection and mapping of galaxy clusters in only a few hours integration time per cluster. Although NIKA2 alone will be a key tool for further understanding cluster physics, using the complementarity between different observational probes constitutes the best road for getting a comprehensive picture of the ICM. The NIKA2 data will therefore be complemented with ancillary data including X-ray, optical, and radio observations. The full data set will lead to significant improvements on the use of galaxy clusters to obtain constraints on cosmology and on the matter distribution and content of the Universe.

Acknowledgements. We would like to thank the IRAM staff for their support during the campaigns. The NIKA dilution cryostat has been designed and built at the Institut Néel. In particular, we acknowledge the crucial contribution of the Cryogenics Group, and in particular Gregory Garde, Henri Rodenas, Jean Paul Leggeri, Philippe Camus. This work has been partially funded by the Foundation Nanoscience Grenoble, the LabEx FOCUS ANR-11-LABX-0013, and the ANR under the contracts "MKIDS", "NIKA", and ANR-15-CE31-0017. This work has benefited from the support of the European Research Council Advanced Grant ORISTARS under the European Union's Seventh Framework Programme (Grant Agreement No. 291294). We acknowledge fundings from the ENIGMASS French LabEx (R.A. and F.R.), the CNES post-doctoral fellowship program (R.A.), the CNES doctoral fellowship program (A.R.), and the FOCUS French LabEx doctoral fellowship program (A.R.).

\section{References}

Adam, R. 2015, Ph.D. Thesis, Université Grenoble Alpes, France Adam, R., Comis, B., Macías-Pérez, J. F., et al. 2014, A\&A, 569, A66 Adam, R., Comis, B., Macías-Pérez, J. F., et al. 2015, A\&A, 576, A12 Adam, R., Comis, B., Bartalucci, I., et al. 2016, A\&A, 586, A122 Adam, R., Bartalucci, I., Pratt, G. W., et al. 2017, A\&A, in press, DOI: 10.1051/0004-6361/201629182

Ameglio, S., Borgani, S., Pierpaoli, E., \& Dolag, K. 2007, MNRAS, 382, 397 Anderson, L., Aubourg, E., Bailey, S., et al. 2014, MNRAS, 441, 24 Applegate, D. E., Douglas, E., von der Linden, A., et al. 2014, MNRAS, 439, 48 Arnaud, M., Pratt, G. W., Piffaretti, R., et al. 2010, A\&A, 517, A92 Basu, K., Zhang, Y.-Y., Sommer, M. W., et al. 2010, A\&A, 519, A29 Becker, R. H., White, R. L., \& Helfand, D. J. 1995, ApJ, 450, 559 Birkinshaw, M. 1999, Phys. Rep., 310, 97

Biviano, A., Murante, G., Borgani, S., et al. 2006, A\&A, 456, 23 Bleem, L. E., Stalder, B., de Haan, T., et al. 2015, ApJS, 216, 27 Böhringer, H., Pratt, G. W., Arnaud, M., et al. 2007, A\&A, 469, 363 Böhringer, H., Pratt, G. W., Arnaud, M., et al. 2010, A\&A, 514, A32 Bonamente, M., Tucker, R. D., Terry, M. P., et al. 2012, New J. Phys., 14, 025010 Bourrion, O., Vescovi, C., Bouly, J. L., et al. 2012, J. Instrum., 7, 7014 Calvo, M., Roesch, M., Désert, F.-X., et al. 2013, A\&A, 551, L12 Carlstrom, J. E., Holder, G. P., \& Reese, E. D. 2002, ARA\&A, 40, 643 Catalano, A., Calvo, M., Ponthieu, N., et al. 2014, A\&A, 569, A9 Catalano, A., Calvo, M., Ponthieu, N., et al. 2016, ArXiv e-print [arXiv: 1605.08628]

Comis, B., Adam, R., Ade, P., et al. 2016, ArXiv e-print [arXiv: 1605. 09549]

Condon, J. J., Cotton, W. D., Greisen, E. W., et al. 1998, AJ, 115, 1693

Croston, J. H., Arnaud, M., Pointecouteau, E., \& Pratt, G. W. 2006, A\&A, 459, 1007 
da Silva, A. C., Kay, S. T., Liddle, A. R., \& Thomas, P. A. 2004, MNRAS, 348, 1401

de Haan, T., Benson, B. A., Bleem, L. E., et al. 2016, ApJ, 832, 95

Démoclès, J., Pratt, G. W., Pierini, D., et al. 2010, A\&A, 517, A52

Donahue, M., Horner, D. J., Cavagnolo, K. W., \& Voit, G. M. 2006, ApJ, 643, 730

Ebeling, H. 2014, Beyond MACS: A Snapshot Survey of the Most Massive Clusters of Galaxies at $z>0.5$, HST Proposal ID 13671, 22

Eckert, D., Ettori, S., Molendi, S., Vazza, F., \& Paltani, S. 2013, A\&A, 551, A23

Gavazzi, R. 2005, A\&A, 443, 793

Gelman, A., \& Rubin, D. B. 1992, Statist. Sci., 7, 457

Griffin, M. J., Abergel, A., Abreu, A., et al. 2010, A\&A, 518, L3

Halverson, N., Lanting, T., Ade, P. A. R., et al. 2009, ApJ, 701, 42

Hasselfield, M., Hilton, M., Marriage, T. A., et al. 2013, JCAP, 1307, 008

Hoekstra, H., Herbonnet, R., Muzzin, A., et al. 2015, MNRAS, 449, 685

Itoh, N., Kohyama, Y., \& Nozawa, S. 1998, ApJ, 502, 7

Kompaneets, A. 1956, Zh. Eksp. Teor. Fiz., 31, 876

Korngut, P. M., Dicker, S. R., Reese, E. D., et al. 2011, ApJ, 734, 10

Lee, J., \& Suto, Y. 2004, ApJ, 601, L599

Mayet, F., Adam, R., Adane, A., et al. 2016, ArXiv e-print [arXiv: 1602.07941]

Mazzotta, P., Rasia, E., Moscardini, L., \& Tormen, G. 2004, MNRAS, 354, 10

Monfardini, A., Benoit, A., Bideaud, A., et al. 2011, ApJS, 194, 24

Moreno, R. 2010, Neptune and Uranus planetary brightness temperature tabulation, Tech. Rep., ESA Herschel Science Center, ftp://ftp.sciops. esa.int/pub/hsc-calibration/PlanetaryModels/ESA2

Muchovej, S., Mroczkowski, T., Carlstrom, J. E., et al. 2007, ApJ, 663, 708

Nagai, D., Kravtsov, A. V., \& Vikhlinin, A. 2007a, ApJ, 668, 1

Nagai, D., Vikhlinin, A., \& Kravtsov, A. V. 2007b, ApJ, 655, 98

Nord, M., Basu, K., Pacaud, F., et al. 2009, A\&A, 506, 623

Perrott, Y. C., Olamaie, M., Rumsey, C., et al. 2015, A\&A, 580, A95

Plagge, T., Benson, B. A., Ade, P. A. R., et al. 2010, ApJ, 716, 1118
Plagge, T. J., Marrone, D. P., Abdulla, Z., et al. 2013, ApJ, 770, 112 Planck Collaboration IX. 2011, A\&A, 536, A9

Planck Collaboration Int. V. 2013, A\&A, 550, A131

Planck Collaboration VIII. 2014, A\&A, 571, A8

Planck Collaboration XXIX. 2014, A\&A, 571, A29

Planck Collaboration XXXII. 2015, A\&A, 581, A14

Planck Collaboration XIII. 2016, A\&A, 594, A13

Planck Collaboration XXII. 2016, A\&A, 594, A22

Planck Collaboration XXIV. 2016, A\&A, 594, A24

Planck Collaboration XXVII. 2016, A\&A, 594, A27

Pratt, G. W., Croston, J. H., Arnaud, M., \& Böhringer, H. 2009, A\&A, 498, 361

Pratt, G. W., Arnaud, M., Piffaretti, R., et al. 2010, A\&A, 511, A85

Puchwein, E., Sijacki, D., \& Springel, V. 2008, ApJ, 687, L53

Riess, A. G., Strolger, L.G., Casertano, S., et al. 2007, ApJ, 659, 98

Sayers, J., Czakon, N. G., Bridge, C., et al. 2012, ApJ, 749, L15

Sayers, J., Czakon, N. G., Mantz, A., et al. 2013, ApJ, 768, 177

Schwan, D., Ade, P. A. R., Basu, K., et al. 2011, Rev. Sci. Instrum., 82, 091301

Sehgal, N., Trac, H., Acquaviva, V., et al. 2011, ApJ, 732, 44

Sembolini, F., Petris, M. D., Yepes, G., et al. 2014, MNRAS, 440, 3520

Sifón, C., Battaglia, N., Hasselfield, M., et al. 2016, MNRAS, 461, 248

Staguhn, J., Allen, C., Benford, D., et al. 2008, J. Low Temperature Phys., 151, 709

Sunyaev, R. A., \& Zeldovich, Y. B. 1972, Comm. Astrophys. Space Phys., 4, 173

Sunyaev, R. A., \& Zeldovich, Ya. B. 1980, ARA\&A, 18, 537

Umetsu, K., Medezinski, E., Nonino, M., et al. 2014, ApJ, 795, 163

Vikhlinin, A. 2006, ApJ, 640, 710

Vikhlinin, A., Kravtsov, A., Forman, W., et al. 2006, ApJ, 640, 691

Voit, G. M. 2005, Rev. Mod. Phys., 77, 207

Voit, G. M., Kay, S. T., \& Bryan, G. L. 2005, MNRAS, 364, 909

Witzel, A., Pauliny-Toth, I. I. K., \& Nauber, U. 1979, AJ, 84, 942

Yu, L., Nelson, K., \& Nagai, D. 2015, ApJ, 807, 12 